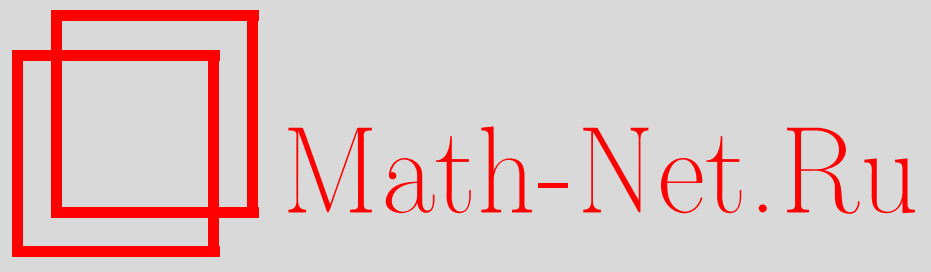

В. В. Белов, В. А. Максимов, Квазиклассическое квантование боровских орбит в атоме гелия, ТМФ, 2007, том 151, номер 2, 261-286

DOI: https://doi.org/10.4213/tmf6044

Использование Общероссийского математического портала Math-Net.Ru подразумевает, что вы прочитали и согласны с пользовательским соглашением http://www . mathnet.ru/rus/agreement

Параметры загрузки:

IP : 52.6 .47 .48

26 апреля 2023 г., 14:40:33

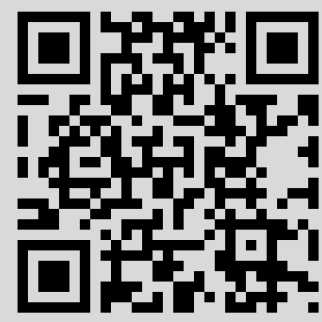




\title{
КВАЗИКЛАССИЧЕСКОЕ КВАНТОВАНИЕ БОРОВСКИХ ОРБИТ В АТОМЕ ГЕЛИЯ
}

\begin{abstract}
На основе комплексного метода ВКБ-Маслова построена квазиклассическая спектральная серия, соответствующая резонансным боровским орбитам в атоме гелия. Квазиклассические уровни энергии, представленные в форме тетраридберговской серии, отвечают дважды симметрично возбужденным состояниям гелиеподобных атомов. Эта серия уровней содержит в себе тройную ридберговскую серию (Рихтер, Винтген, 1991), отвечающую $Z^{2+} e^{-} e^{-}$-конфигурации электронов, которая наблюдалась в экспериментах Эйхмана с сотрудниками по лазерному возбуждению атома бария (1992 г.). Экстраполяция на нижние уровни полученной формулы для квазиклассического спектра дает значение энергии основного состояния, отличающееся на $6 \%$ от экспериментального (Бергесон с сотрудниками, 1998). Также вычислена тонкая структура квазиклассического спектра, обусловленная спин-орбитальным и спин-спиновым взаимодействиями электронов.
\end{abstract}

Ключевые слова: квазиклассическое квантование, спектральные серии, комплексный метод ВКБ-Маслова, обобщенные переменные действие-угол и гармонического осциллятора.

\section{1. ВВЕДЕНИЕ И ФОРМУЛИРОВКА РЕЗУЛЬТАТОВ}

В 1913 г. во второй части своей знаменитой серии работ [1], заложивших основы квантовой механики, Нильс Бор проквантовал периодическое коррелированное движение в атоме гелия (движение электронов вдоль равновесной окружности $S^{1}(E)$ радиуса $R=R(E)$, где $E<0$ - классическая энергия системы, см. ниже рис. 1).

Оказалось, однако, что формулы квазиклассического квантования, основанные на постулатах Бора (сейчас их часто называют формулами "старой" квантовой теории), успешно воспроизводившие спектр атома водорода, не дают удовлетворительных результатов при вычислении как основного, так и возбужденных состояний атома гелия (детали см., например, в обзоре [2]).

Использование квазиклассического приближения, основанного на более общих формулах квантования типа формул Бора-Зоммерфельда, и применение математической версии этого приближения (теории канонического оператора Маслова с

* Московский государственный институт электроники и математики, Москва, Россия. E-mail: pm@miem.edu.ru 
вещественной фазой [3], [4]) также не меняют ситуацию. Как отмечает в своей известной книге [5] Лере, квантование Маслова дает результат “старой” квантовой теории, сохраняя простоту вычислений; это квантование, возникающее из чисто математических рассуждений, может заменить квантование Бора-Зоммерфельда. С физической точки зрения такая замена будет оправданна, если она окажется применимой к атому с несколькими электронами.

Простейшей моделью многоэлектронной системы является модель гелиеподобного атома (иона) - два электрона в поле заряженного неподвижного центра с гамильтонианом

$$
\begin{aligned}
& H(p, q)=\frac{1}{2 m}\left(\left|p_{1}\right|^{2}+\left|p_{2}\right|^{2}\right)+V\left(q_{1}, q_{2}\right), \\
& p=\left(p_{1}, p_{2}\right) \in \mathbb{R}_{p}^{6}, \quad q=\left(q_{1}, q_{2}\right) \in \mathbb{R}_{q}^{6},
\end{aligned}
$$

где $V\left(q_{1}, q_{2}\right)$ - потенциальная энергия кулоновского взаимодействия между двумя электронами, $q_{i}=\left(x_{i}, y_{i}, z_{i}\right), i=1,2$, и неподвижным ядром с зарядом $Z(Z \geqslant 2)$, помещенным в начало координат,

$$
V\left(q_{1}, q_{2}\right)=-\frac{Z e^{2}}{\left|q_{1}\right|}-\frac{Z e^{2}}{\left|q_{2}\right|}+\frac{e^{2}}{\left|q_{1}-q_{2}\right|},
$$

$e=-e_{0}$ и $m-$ заряд и масса электрона.

Цель этой работы - проквантовать (в квазикласическом приближении) семейство боровских орбит $S^{1}(E)$ в атоме гелия.

Это означает, что для соответствующей (1) спектральной квантовой задачи $\widehat{H} \Psi=$ $E \Psi$ с оператором Шредингера $\widehat{H}=H(-i \hbar \partial / \partial q, q)$, где $\hbar$ - "малый параметр", $\hbar \in$ $(0,1)$, требуется построить последовательность чисел $E_{\nu}(\hbar)$ (квазиклассические уровни энергии) и последовательность функций $\Psi_{\nu}(q, \hbar) \in L_{2}\left(\mathbb{R}_{q}^{6}\right)$ (отвечающие $E_{\nu}(\hbar)$ квазиклассические волновые функции), которые при $\hbar \rightarrow 0$ удовлетворяют условиям

$$
\left(\widehat{H}-E_{\nu}(\hbar)\right) \Psi_{\nu}(q, \hbar)=O\left(\hbar^{\alpha}\right), \quad\left\|\Psi_{\nu}\right\|=1 .
$$

Здесь $\alpha=3 / 2, \nu=\left(\nu_{1}, \nu_{2}, \ldots, \nu_{r}\right) \in \mathbb{Z}_{+}^{r}$ - мультииндекс, нумерующий асимптотические собственные значения $E_{\nu}(\hbar), 1 \leqslant r \leqslant 6, O\left(\hbar^{\alpha}\right)$ - оценка невязки уравнения в норме $L_{2}\left(\mathbb{R}_{q}^{6}\right)$. При этом $\Psi_{\nu}$ и $E_{\nu}(\hbar)$ локализованы в окрестности боровских орбит в том смысле, что

$$
\lim _{\hbar \rightarrow 0} \operatorname{supp} \Psi_{\nu}=S^{1}(E), \quad \lim _{\hbar \rightarrow 0} E_{\nu}(\hbar)=E .
$$

Число $E_{\nu}(\hbar)$ и функцию $\Psi_{\nu}$, удовлетворяющие условиям $(3)$, часто называют [6] $\kappa в a-$ зимодой, а последовательность $\left(E_{\nu}(\hbar), \Psi_{\nu}\right)$, удовлетворяющую $(3),(4),-$ спектральной серией, отвечающей в пределе при $\hbar \rightarrow 0$ движению классической системы по боровской орбите $S^{1}(E)$.

ЗАмЕчАНИЕ 1. Процесс построения квазимод в квантовой физике - это процесс некоторых “приближенных вычислений”, в котором постоянная Планка $ћ$ рассматривается как бесконечно малая, при этом не сравнивается порядок численных значений 
рассматриваемых величин, когда $\hbar$ принимает свое истинное физическое значение. Это значение придается $\hbar$ только после завершения вычислений, предполагающих $\hbar$ бесконечно малой. Оправданием такой формальной процедуры являются численные результаты, которые согласуются с экспериментом. Выбор реального безразмерного параметра квазиклассических разложений в каждой физической проблеме - это отдельная, подчас нетривиальная задача. Для одномерных квантовомеханических задач таким параметром (пропорциональным $\hbar$ ) принято считать отношение длины волны де Бройля частицы к характерным размерам системы. Для рассматриваемой здесь задачи построения квазиклассических асимптотик, локализованных в окрестности боровских орбит, безразмерные параметры квазиклассического разложения, пропорциональные $\hbar$ и $\sqrt{\hbar}$, будут указаны ниже (см. раздел 2 ).

Коррелированному движению двух электронов по окружности $S^{1}(E)$ в плоскости $z=0$ физического пространства $\mathbb{R}_{x, y, z}^{3}$ соответствует замкнутая фазовая траектория $\Lambda^{1}(E)$ гамильтоновой системы с гамильтонианом (1) в фазовом 12-мерном пространстве $\mathbb{R}_{p, q}^{12}$ классической системы. При этом сама боровская орбита $S^{1}(E)$ есть результат проектирования $\Lambda^{1}(E)$ на конфигурационное пространство системы $\mathbb{R}^{6}=\mathbb{R}_{q_{1}}^{3} \times \mathbb{R}_{q_{2}}^{3}$ с последующим отождествлением точек двух трехмерных гиперплоскостей с координатами $\left(x_{1}, y_{1}, z_{1}, 0,0,0\right)$ и $\left(0,0,0, x_{2}, y_{2}, z_{2}\right)$ с точкой $(x, y, z)$ физического пространства $\left(x_{i}=x, y_{i}=y, z_{i}=z, i=1,2\right)$.

Отметим теперь, что замкнутые фазовые кривые явлются частным и наиболее простым (наряду с точками покоя) случаем инвариантных неполномерных лагранжевых (изотропных) торов $\Lambda^{k}(k<n, n$ - размерность конфигурационного пространства) гамильтоновых систем. Напомним, что $k$-мерный тор $\Lambda^{k}$ называется изотропным, если на нем аннулируется каноническая 2-форма $d p \wedge d q$. Такие торы являются первым из основных геометрических объектов в теории квазиклассического квантования, развитой относительно недавно в работах [7]-[10]. Эта теория (комплексный метод ВКБ, Вентцель [11]-Крамерс [12]-Бриллюэн [13]), основанная на понятии комплексного ростка Маслова, позволяет сопоставить инвариантным маломерным торам $\Lambda^{k}$ классической (гамильтоновой) системы спектральные серии $\left(E_{\nu}(\hbar), \Psi_{\nu}\right)$ соответствующей квантовой системы или, другими словами, прокванmовать $\Lambda^{k}$ в квазиклассическом приближении. В отличие от полномерного случая $(k=n)$ проквантовать $\Lambda^{k}$ при $k<n$ можно лишь при некоторых дополнительных условиях на торы $\Lambda^{k}$ (см. [7]), наиболее существенным из которых является требование их орбитальной устойчивости (в линейном приближении).

ЗАмечАние 2. Это требование (в случае $k=1$ ) содержится уже в пионерской работе Бабича [14] (для замкнутой геодезической) и в работах [15], [16] (для замкнутых траекторий общих гамильтонианов).

С геометрической точки зрения свойство орбитальной устойчивости означает, что может быть построено векторное расслоение $\left[\Lambda^{k}, r^{n}\right]$ с базой $\Lambda^{k}$ и слоями $r^{n}-$ некоторыми $n$-мерными плоскостями в комплексном фазовом пространстве; причем эти 
слои обладают свойством инвариантности относительно системы в вариациях - линеаризации исходной гамильтоновой системы в окрестности инвариантного изотропного тора $\Lambda^{k}$. Существование таких плоскостей, называемых комплексным ростком Маслова, является необходимым условием для построения квазимод, соответствующих $k$-мерным торам, $0 \leqslant k<n$. В случаях $k=0$ и $k=1$ это условие является и достаточным.

ЗАмечАниЕ 3. Квазимоды оператора $\widehat{H}$ дают информацию об истинных модах - точных собственных функциях $\Psi_{\nu}^{\mathrm{ex}}(q, \hbar)$ и собственных значениях $E_{\nu}^{\mathrm{ex}}(\hbar)$ этого оператора. В силу оценки (3) и известной леммы функционального анализа (см., например, [3]) расстояние между $E_{\nu}(\hbar)$ и спектром оператора $\widehat{H}$ есть $O_{\nu}\left(\hbar^{\alpha}\right), \alpha>1$. Иными словами в $O\left(\hbar^{\alpha}\right)$-окрестности точки $E_{\nu}(\hbar)$ при $\hbar \rightarrow 0$ найдется хотя бъ одно собственное значение $E_{\nu}^{\mathrm{ex}}$ такое, что $\left|E_{\nu}(\hbar)-E_{\nu}^{\mathrm{ex}}(\hbar)\right| \leqslant C \hbar^{\alpha}(C>0$ - константа). При этом асимптотические собственные функции $\Psi_{\nu}(q, \hbar)$ близки к некоторой линейной комбинации точных собственных функций.

Приведем теперь, следуя [10], условия квантования замкнутых фазовых траекторий с комплексным ростком (условия квантования типа Бора-ЗоммерфельдаМаслова), которые определяют в первом приближении по $\hbar \rightarrow 0$ серию асимптотических собственных значений $E_{\nu}(\hbar)$. Соответствующие $E_{\nu}(\hbar)$ квазиклассические волновые функции $\Psi_{\nu}(q, \hbar)$ можно получить, если использовать комплексный канонический оператор Маслова [7] (см. также [8]).

Пусть в $2 n$-мерном евклидовом фазовом пространстве $\mathbb{R}_{p, q}^{2 n}$ гамильтонова система

$$
\dot{p}=-\nabla_{q} H, \quad \dot{q}=\nabla_{p} H \quad \Longleftrightarrow \quad \dot{y}=J H_{y}
$$

где $H=H(y)=H(p, q) \in C^{\infty}\left(\mathbb{R}_{p, q}^{2 n}\right)$ - функция Гамильтона, $y=(p, q)$, имеет гладкое однопараметрическое семейство замкнутых траекторий $\Lambda^{1}(E)$. Здесь параметр $E-$ классическая энергия, $E \in M_{E}, M_{E}$ - интервал на $\mathbb{R}_{E}^{1}, J$ - стандартная симплектическая $(2 n \times 2 n)$-матрица $J=\left(\begin{array}{cc}0 & -E_{n} \\ E_{n} & 0\end{array}\right), E_{n}$ - единичная $(n \times n)$-матрица.

Для дальнейших рассуждений удобно параметризовать семейство $\Lambda^{1}(E)$ переменной действия $I=I(E)$, где $I(E)=(2 \pi)^{-1} \oint_{\Lambda^{1}(E)}\langle p, d q\rangle$, сопряженной угловой переменной $\varphi(\bmod 2 \pi)$. Здесь $\langle\cdot, \cdot\rangle$ - скалярное произведение в $\mathbb{R}^{3}$. Тогда

$$
\Lambda^{1}(I)=\Lambda^{1}(E(I))=\left\{y, y=Y\left(\omega_{0} t+\varphi, I\right)=\left(P\left(\omega_{0} t+\varphi, I\right), Q\left(\omega_{0} t+\varphi, I\right)\right)\right\},
$$

где $P(\varphi, I), Q(\varphi, I)$ - гладкие $2 \pi$-периодические вектор-функции, $\omega_{0}=\omega_{0}(I)=$ $\left.\frac{\partial}{\partial I} H\right|_{\Lambda^{1}(I)}$ - частота периодического движения системы вдоль фазовой кривой $\Lambda^{1}(I)$, $I \in M_{I}, M_{I}-$ интервал на $\mathbb{R}_{I}^{1}$.

Гамильтониан $H(p, q)$ и траектория $\Lambda^{1}(I)$ порождают линеаризованную в окрестности $\Lambda^{1}(I)$ гамильтонову систему - систему в вариациях

$$
\dot{v}=J H_{y y}\left(Y\left(\omega_{0} t+\varphi, I\right)\right) v, \quad v \in \mathbb{C}^{2 n} .
$$

Очевидно, что вектор-функции $Y_{\varphi}^{\prime}=\frac{\partial Y}{\partial \varphi}\left(\omega_{0} t+\varphi, I\right)$ и $Y_{I}^{\prime}=\frac{\partial Y}{\partial I}\left(\omega_{0} t+\varphi, I\right)$ являются решениями этой системы. Орбитальная устойчивость траекторий $\Lambda^{1}(I)$ (условие существования комплексного ростка $r^{n}(I)$ на $\left.\Lambda^{1}(I)\right)$ означает тогда, что базис решений 
Флоке системы (7), гладко зависящих от $I \in M_{I}$ и косоортогональных решениям $Y_{\varphi}^{\prime}$ и $Y_{I}^{\prime}$, имеет вид $\left\{v_{j}(t), \bar{v}_{j}(t), j=2,3, \ldots, n\right\}, v_{j}(t, I)=V_{j}\left(\omega_{0} t+\varphi, I\right) e^{i \beta_{j} t}$, где показатели Флоке $\beta_{j}=\beta_{j}(I)$ - вещественные числа. Здесь $V_{j}(\varphi, I)-2 \pi$-периодические вектор-функции аргумента $\varphi$.

Как показано в [17], [18], в полной трубчатой окрестности $U_{\delta}$ орбитально устойчивой фазовой кривой $\Lambda^{1}(I), I \in M_{I}$, можно ввести такие обобщенные канонические переменные типа действие-угол (переменные действие-угол $(J, \varphi)$ и переменные $(n-1)$-мерного гармонического осциллятора $\bar{y}=(\bar{p}, \bar{x}), \bar{p}=\left(\bar{p}_{2}, \ldots, \bar{p}_{n}\right)$, $\left.\bar{x}=\left(\bar{x}_{2}, \ldots, \bar{x}_{n}\right)\right)$, явно задаваемые с помощью системы квадратных уравнений, что исходный гамильтониан $H$ в этих переменных в разложении по $\bar{y},\|\bar{y}\|<\delta, \delta>0-$ постоянная, (равномерно по $\varphi$ ) имеет вид

$$
H=H(y(J, \varphi, \bar{y}))=H_{2}(I, \bar{y})+O\left(\|\bar{y}\|^{3}\right), \quad\|\bar{y}\| \rightarrow 0,
$$

где $H_{2}$ - нормальная форма гамильтониана $H$ (в квадратичном приближении) в окрестности кривой $\Lambda^{1}(I)$ :

$$
H_{2}(I, \bar{y})=\left.H\right|_{\Lambda^{1}(I)}+\sum_{j=2}^{n} \beta_{j}(I)\left(\frac{\bar{p}_{j}^{2}+\bar{x}_{j}^{2}}{2}\right), \quad I \in M_{I}, \quad\|\bar{y}\|<\delta .
$$

Условия квантования гладкого семейства замкнутых фазовых кривых $\Lambda^{1}(I)$ с комплексным ростком $r^{n}(I)$ есть условие квантования параметра семейства $I$, т.е. переменной действия $I$ на $\Lambda^{1}(I)$ и переменных действия $\rho=\left(\rho_{2}, \rho_{3}, \ldots, \rho_{n}\right)$ гармонического осциллятора, $2 \rho_{j}=\bar{p}_{j}^{2}+\bar{x}_{j}^{2}$. Это условие состоит в выборе при каждом фиксированном $\hbar, 0<\hbar<\hbar_{0}$, где $\hbar_{0}$ достаточно мало, дискретного набора значений $I^{\nu_{1}}(\hbar) \in M_{I}, \rho^{\bar{\nu}}(\hbar)=\left(\rho_{2}^{\nu_{2}}(\hbar), \ldots, \rho_{n}^{\nu_{n}}(\hbar)\right) \in\{\rho,\|\rho\|<\delta / 2\}$ в соответствии с формулами

$$
\begin{array}{ll}
I^{\nu_{1}}(\hbar)=\hbar\left(\nu_{1}+\frac{\sigma}{4}\right), & \nu_{1} \in \mathbb{Z}, \\
\rho_{j}^{\nu_{j}}(\hbar)=\hbar\left(\nu_{j}+\frac{1}{2}\right), & \nu_{j} \in \mathbb{Z}^{+}, \quad j=2, \ldots, n,
\end{array}
$$

где $\sigma \in \mathbb{Z}$ - индекс комплексного ростка $r^{n}(I)$ на кривой $\Lambda^{1}(I)$ (явные формулы для вычисления индекса $\sigma$ приведены в [10]).

Квантованные значения обобщенных переменных действия $I^{\nu_{1}}(\hbar), \rho_{j}^{\nu_{j}}(\hbar), j=$ $2, \ldots, n$, определяют спектральную серию квазиклассических уровней энергии исходного оператора $\widehat{H}$, отвечающую семейству $\left\{\Lambda^{1}\left(I^{\nu_{1}}(\hbar)\right), r^{n}\left(I^{\nu_{1}}(\hbar)\right)\right\}$, по формуле

$$
E_{\nu}(\hbar)=E_{\nu_{1}, \bar{\nu}}(\hbar)=\left.H\right|_{\Lambda^{1}\left(I^{\nu_{1}}(\hbar)\right)}+\sum_{j=2}^{n} \beta_{j}\left(I^{\nu_{1}}(\hbar)\right)\left(\nu_{j}+\frac{1}{2}\right) .
$$

Для $k$-параметрического гладкого семейства изотропных торов $\Lambda^{k}(I)$ с комплексным ростком $r^{n}(I), I=\left(I_{1}, I_{2}, \ldots, I_{k}\right) \in M_{I}, M_{I}$ - открытая область в $\mathbb{R}_{I}^{k}$, аналогичная формула имеет место при следующих дополнительных условиях: 
1) условие нерезонансности: часто́ты условно периодического движения $\omega_{j}(I)$, $j=1,2, \ldots, k$, на торе $\Lambda^{k}(I)$ при $I=I^{\nu}(h)$ диофантово несоизмеримы, т.е.

$$
\sum_{j=1}^{k} \omega_{j}(I) m_{j}=(\omega(I), m) \geqslant C|m|^{-r}, \quad C>0, \quad r>0, \quad m \in \mathbb{Z}^{k}
$$

2) соответствующая система в вариациях с квазипериодическими коэффициентами является приводимой [19] с (вещественнъми) числами вращения $\beta_{j}(I), j=$ $1, \ldots, k$ (аналогами показателей Флоке).

При этих условиях серия квазиклассических уровней энергии, отвечающая семейству $\left\{\Lambda^{k}(I), r^{n}(I)\right\}$, определяется по формуле, аналогичной (10):

$$
E_{\nu}(\hbar)=\left.H\right|_{\Lambda^{k}\left(I^{\nu}(\hbar)\right)}+\hbar \sum_{j=n-k-1}^{n} \beta_{j}\left(I^{\nu}(\hbar)\right)\left(\nu_{j}+\frac{1}{2}\right),
$$

где $I^{\nu}(\hbar)$ - квантованные значения переменных действия на торе $\Lambda^{k}(I), I^{\nu}(\hbar) \in M_{I}$, определяемые в соответствии со следующей формулой квантования:

$$
I_{j}^{\nu}(\hbar)=\hbar\left(\nu_{j}+\frac{\sigma_{j}}{4}\right), \quad \nu_{j} \in \mathbb{Z}, \quad j=1,2, \ldots, k,
$$

где $\sigma_{j}$ - индекс комплексного ростка $j$-го базисного цикла на изотропном торе $\Lambda^{k}(I)$.

Заметим теперь, что существование неполномерных (или маломерных) инвариантных лагранжевых торов $\Lambda^{k}$ и даже их $k$-мерных семейств не предполагает интегрируемости исходной гамильтоновой системы. Более того, часто в неинтегрируемом случае, к которому относится гамильтониан атома гелия (1), только такие маломерные инвариантные объекты и удается явно построить. В этой ситуации для квантовых спектральных задач с классически неинтегрируемым гамильтонианом комплексный метод ВКБ позволяет получить (в квазиклассическом приближении) информацию о спектре квантовой системы. Примеры таких квантовых систем с одночастичным квантовым гамилътонианом (учитывающим спин электрона) можно найти в работах [20], [21] для модели нелинейных двух связанных осцилляторов с потенциалом $V(x, y)=\mu\left(x^{4}+y^{4}\right)+x^{2} y^{2}, \mu>0$; в работах [22], [23] для анизотропной квантовой задачи Кеплера; в работе [24] для атома водорода в однородном магнитном поле; в работах [25] для анизотропного атома водорода с учетом спина электрона в однородном магнитном поле; в работе [26] для трехмерного ангармонического осциллятора с переменной частотой. В этих работах получены новые явные (аналитические) формулы квазиклассических спектральных серий, отвечающих специальным семействам изотропных торов $\Lambda^{k}$ при $k=1,2$.

Разумеется, инвариантные изотропные торы $\Lambda^{k}, k<n$, возникают и для интегрирумых (по Лиувиллю) гамильтоновых систем. Типичной является ситуация, когда лиувиллевы $n$-мерные лагранжевы торы $\Lambda^{n}$ оказываются резонансными. При резонансе частот условно периодического движения по тору $\Lambda^{n}$ траектории соответствующей гамильтоновой системы могут образовывать торы $\Lambda^{k} \subset \Lambda^{n}$ меньшей 
размерности $(1 \leqslant k<n)$ [27]. Другой важный случай связан с вырождением переменных действие-угол, существование которых в интегрируемом случае гарантируется теоремой Лиувилля [28]. Переменные действия, как правило, не являются глобальными во всем фазовом пространстве. Оно разбивается на области, в каждой из которых вводятся “свои” переменные действие-угол. Границам этих областей (там, где нарушены условия теоремы Лиувилля ) соответствуют уже не лиувиллевы торы, а другие геометрические объекты, в частности, в гладком случае - "граничные" торы $\Lambda^{k}$ меньшей размерности $k(k<n)$.

$\mathrm{C}$ квантовомеханической точки зрения такое вырождение в интегрируемых системах обычно не рассматривается. Дело в том, что в интегрируемом случае теория квазиклассического квантования инвариантных (полномерных) лагранжевых торов $\Lambda^{k}$ в случае $k=n$ (теория ЭБК (Эйнштейн [29]-Бриллюэн [13]-Келлер [30])ВКБ-Маслова, или “полномерная" теория квазиклассического квантования) требует при определении квазиклассических уровней энергии $E_{\nu}(\hbar)$ знания не траектории неквантованного электрона, а лишь значений первых интегралов системы Гамильтона, определяющих эту траекторию. Условия квантования Бора-ЗоммерфельдаМаслова [31], [3], другими словами, условия квантования значений этих интегралов движения, параметризующих торы $\Lambda^{n}$ (точнее, переменных действия $I=\left(I_{1}, \ldots, I_{n}\right)$, однозначно с ними связанных), всегда определяют в первом приближении по $\hbar$, $\hbar \rightarrow 0$, (возбужденные) уровни энергии $E_{\nu}(\hbar)$ в области больших квантовых чисел $\nu_{j} \sim 1 / \hbar, j=1,2, \ldots, n$.

Для упомянутых выше резонансных торов эти уровни оказываются асимптотически (с точностью $O\left(\hbar^{2}\right)$ ) вырожденными. Амплитуда квазиклассических волновых функций $\Psi_{\nu}(q, \hbar)$, соответствующих $E_{\nu}(\hbar)$, пропорциональна плотности инвариантной меры на $\Lambda^{n}$ [4], которая в этом случае определяется не единственным образом (с точностью до произвольной функции $(n-k)$ угловых переменных на $\Lambda^{n}$, "трансверсальных" $\left.\Lambda^{k} \subset \Lambda^{n}\right)$. Это асимптотическое $\left(\bmod \hbar^{2}\right)$ "бесконечнократное" вырождение снимается, по всей видимости, при построении высших приближений асимптотики $^{1)}$, хотя строгие результаты в этой трудной (при $n \geqslant 2$ ) проблеме до сих пор отсутствуют. В одномерном случае для оператора Шредингера с потенциалами специального вида (в том числе степенны́ми) "точные” условия квантования, дающие квазиклассический спектр с любой степенно́й точностью по параметру $\hbar$, изучались в [32].

Для "граничных" изотропных торов $\Lambda^{k}$ с комплексным ростком $r^{n}$ отвечающие им серии квазиклассических уровней энергии получаются из "полномерных" асимптотик спектра предельным переходом к малым значениям (порядка $O(1), \hbar \rightarrow 0$ ) некоторых чисел из полного набора квантовых чисел $\nu=\left(\nu_{1}, \ldots, \nu_{n}\right)$. Этот набор целых чисел, возникающих в процедуре квантования $n$-мерных лагранжевых торов $\Lambda^{n}$ (в силу условий квантования Бора-Зоммерфельда-Маслова), нумерует торы $\Lambda^{n}=\Lambda^{n}\left(\nu_{1}, \ldots, \nu_{n}\right)$ и, тем самым, отвечающие им квазимоды $\left(E_{\nu}(\hbar), \Psi_{\nu}(q, \hbar)\right)$.

1) Подчеркнем, что речь здесь идет о степенно́м по параметру $\hbar$ вырождении, а не об экспоненциально малом расщеплении собственных значений, обусловленном туннельными эффектами. 
Например, если вырождение торов $\Lambda^{n}\left(I_{1}, I_{2}, \ldots, I_{n}\right)$ в "граничные" изотропные торы $\Lambda^{k}, \Lambda^{k}=\Lambda^{k}\left(I_{1}, \ldots, I_{k}\right)$, происходит по переменным действия $I_{k+1}, \ldots, I_{n}$ (при их стремлении к нулю), то малыми квантовыми числами $\nu_{j}, \nu_{j} \sim 1$, естественно, будут числа с номерами $j=k+1, \ldots, n$. Аккуратное доказательство этого факта при некоторых дополнительных условиях (см. условия 1 и 2 ) на торы $\Lambda^{k}$ приведено в [10] (ср. с методом, приведенным в [33], для замкнутых траекторий).

В формулах квантования (9)-(12) комплексного метода ВКБ в этом случае числа вращения $\beta_{j}=\beta_{j}\left(I_{1}, \ldots, I_{k}\right), j=k+1, \ldots, n$, для тора $\Lambda^{k}\left(I_{1}, \ldots, I_{k}\right)$ есть "предельные осцилляторные частоты" для частот $\omega_{j}\left(I_{1}, \ldots, I_{n}\right)$ условно периодического движения на торе $\Lambda^{n}\left(I_{1}, \ldots, I_{k}, I_{k+1}, \ldots, I_{n}\right)$, т.е. $\beta_{j}=\omega_{j}\left(I_{1}, I_{2}, \ldots, I_{k}, 0,0, \ldots, 0\right)$.

При этом, разумеется, такой предельный переход не имеет места для асимптотических собственных функций, отвечающих изотропным торам с комплексным ростком: их структура совершенно отлична от структуры "полномерных" квазиклассических собственных функций, задаваемых каноническим оператором Маслова.

Проблема резонансов в теории квазиклассического квантования, о которой мы говорили в связи с "полномерным" вариантом этой теории, возникает и при квантовании изотропных торов, если частоты $\omega_{j}(I), j=1, \ldots, k$, на торе $\Lambda^{k}(I)$ соизмеримы или плохо несоизмеримы (т.е. условие 1 не выполнено). Эта резонансная ситуация для неинтегрируемых систем обсуждалась с разных точек зрения в работах [23], [34]. Один из возможных подходов (еще далекий от завершения) к квантованию резонансных изотропных торов $\Lambda^{k}$ с комплексным ростком $r^{n}$ предложен в [10]. Отметим, что наряду с резонансом частот движения на торе $\Lambda^{k}$, приводящих к асимптотическому вырождению серий соответствующих собственных значений $E_{\nu}(\hbar)$, это вырождение может быть связано также с резонансом по "ростковым" переменным, приводящим к тому, что комплексный росток $r^{n}(I)$ на изотропном торе $\Lambda^{k}(I)$ (даже нерезонансном) определяется неединственным образом. С этой ситуацией мы сталкиваемся, в частности, при квантовании боровской орбиты в атоме гелия (см. раздел 2). Проблема неединственности комплексного ростка изучена в [16] лишь для наиболее простых случаев, когда $k=0$ и $k=1$. В случае $k=1$ для резонансной замкнутой траектории $\Lambda^{1}(I)$ неединственность квазиклассических волновых функций $\Psi_{\nu}(q, \hbar)$, отвечающих энергии $E_{\nu}(\hbar)$, связана с тем, что они приобретают зависимость от непрерывных комплексных параметров, число которых, в свою очередь, зависит от числа частот $\beta_{j}$, попавших в резонанс с частотой движения $\omega_{0}(I)$ вдоль траектории $\Lambda^{1}(I)$. Порядок резонанса, по всей видимости, определяет порядок разложения, в котором это вырождение может быть снято. В частности, замкнутые фазовые траектории $\Lambda^{1}(I)$ в интегрируемых системах всегда являются резонансными (см. [19]), так что число комплексных параметров, входящих в соответствующую волновую функцию, равно $n-1$.

Продемонстрируем все вышесказанное на примере атома водорода - классической интегрируемой по Лиувиллю системы с функцией Гамильтона

$$
H=H(p, q)=\frac{p^{2}}{2 m}-\frac{Z e_{0}^{2}}{|q|}, \quad p \in \mathbb{R}_{p}^{3}, \quad q=(x, y, z) \in \mathbb{R}_{q}^{3}
$$


Подчеркнем, что знаменитое правило квантования Бора “старой” квантовой механики было сформулировано в [1] по существу для (квантования) специального вида замкнутых фазовых траекторий $\Lambda^{1}(E)$ (одномерных инвариантных изотропных торов) этой трехмерной классической системы. В учебниках по квантовой механике принято говорить о квантовании круговых орбит $S^{1}(E)$ электрона, движущегося вокруг ядра в плоскости $z=0$ (рис. 1a), которые являются проекциями фазовых (боровских) траекторий $\Lambda^{1}(E)$ на конфигурационное пространство.
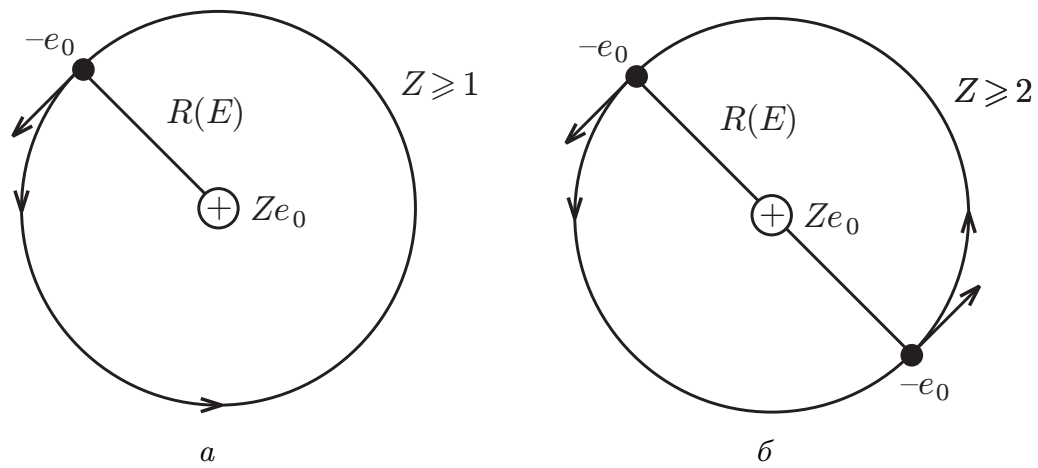

Рис. 1. Боровские орбиты в атоме водорода (а) и атоме гелия (б).

На языке теории гамильтоновых систем с симметриями боровская фазовая траектория $\Lambda^{1}(E)$ представляет собой траекторию стационарного вращения или относительное положение равновесия (ОПР) (см., например, [28]) гамильтоновой системы с циклической переменной. Инвариантность кулоновского потенциала относительно вращения на угол $\varphi$ вокруг, например, оси $z$ порождает соответствующий первый интеграл системы - момент импульса $L_{z}(p, q)=y p_{x}-x p_{y}$ относительно этой оси. Это позволяет стандартным образом [35] осуществить редукцию исходной системы с гамильтонианом $H(13)$ к гамильтоновой системе с двумя степенями свободы на приведенном фазовом пространстве. Точки покоя приведенного гамильтониана $H_{I}$, зависящего от значения $I$ интеграла момента $L_{z}(p, q)=I$ как от параметра, и определяют траектории стационарного вращения для исходной системы. Для рассматриваемого примера переход к приведенному фазовому пространству и приведенному гамильтониану состоит в исключении циклической переменной $\varphi$, которое удобно провести, например, в полярной системе координат $\rho, z, \varphi$ на $\mathbb{R}_{x}^{3}$. Обозначим канонически сопряженные этим координатам импульсы через $p_{\rho}, p_{z}, p_{\varphi}$, где $p_{\varphi}=L_{z}$. Тогда приведенный гамильтониан $H_{I}$ на приведенном фазовом пространстве с координатами $\left(p_{\rho}, p_{z}\right)=\bar{p},(\rho, z)=\bar{q}$ имеет вид

$$
H_{I}=H_{I}(\bar{p}, \bar{q})=\frac{1}{2 m}\left(p_{\rho}^{2}+p_{z}^{2}\right)+V_{I}(\rho, z),
$$

где “эффективный” потенциал

$$
V_{I}(\rho, z)=\frac{I^{2}}{2 m \rho^{2}}-\frac{Z e_{0}^{2}}{\sqrt{\rho^{2}+z^{2}}} .
$$


Точка покоя $\bar{r}(I)=(\bar{p}(I), \bar{q}(I))=(0,0, \rho=R(I), 0)$ функции $H_{I}$, где $R(I)=I^{2} /\left(e_{0}^{2} m\right)$, задает на совместной поверхности уровня интегралов движения $H(p, q)=E$, $L_{z}(p, q)=I$ траекторию $\Lambda^{1}(I)$ исходной гамильтоновой системы по формуле

$$
\Lambda^{1}(I)=\left\{p_{\rho}=0, p_{z}=0, p_{\varphi}=I, \quad \rho=R(I), \quad z=0, \varphi(\bmod 2 \pi)\right\}
$$

при условии, что параметры $E$ и $I, I \neq 0$, связаны соотношением

$$
E=\left.H\right|_{\Lambda^{1}(I)}=H_{I}(\bar{r}(I))=-\frac{\alpha}{I^{2}}, \quad \alpha=\frac{m e_{0}^{4}}{2} .
$$

Очевидно, что проекция $\Lambda^{1}(I)$ на $\mathbb{R}_{q}^{3}$ является окружностью $S^{1}(E)$ (рис. 1б) в плоскости $z=0$ радиуса $R(E)=I^{2} /\left(e_{0}^{2} m\right)$, по которой электрон вращается с частотой

$$
\omega_{0}=\omega_{0}(I)=\frac{\partial}{\partial I} H_{I}(\bar{r}(I))=\frac{m e_{0}^{4}}{|I|^{3}} .
$$

Точка покоя $\bar{r}(I)$ приведенного гамильтониана устойчива в линейном приближении равномерно по $I \in \mathbb{R} \backslash\{0\}$ : частоты нормальных колебаний, радиальных $\omega_{\rho}$ и аксиальных $\omega_{z}$, совпадают с частотой вращения $\omega_{0}(I)$. Отсюда следует, что боровская фазовая траектория $\Lambda^{1}(I)$ орбитально устойчива в линейном приближении, причем (вещественные) показатели Флоке решений системы в вариациях (7), отвечающей $H(13)$ и $\Lambda^{1}(I)(16)$, равны соответственно $\beta_{2}(I)=\omega_{\rho}=\omega_{0}, \beta_{3}(I)=\omega_{z}=\omega_{0}$. Таким образом, в рассматриваемом примере нормальная форма $\left.{ }^{2}\right) H_{2}$ гамильтониана $H(13)$ в окрестности траектории $\Lambda^{1}(I)$ с учетом (17) определяется квадратичной частью разложения в ряд Тейлора приведенного гамильтониана $H_{I}$ по степеням малых отклонений от "равновесных" значений $\rho=R(I)$ и $z=0$. В координатах $\bar{x}_{1}=(\rho-R(I)) / \sqrt{\omega_{0}}, \bar{x}_{2}=z / \sqrt{\omega_{0}}$ и $\bar{p}_{1}=p_{\rho} \sqrt{\omega_{0}}, \bar{p}_{2}=p_{z} \sqrt{\omega_{0}}$ нормальная форма $H_{2}$ гамильтониана $H(13)$ в окрестности боровской фазовой траектории $\Lambda^{1}(I)(16)$ имеет вид

$$
H_{2}(I, \bar{y})=-\frac{m e^{4}}{2 I^{2}}+\frac{m e^{4}}{|I|^{3}}\left(\frac{\bar{x}_{1}^{2}+\bar{p}_{1}^{2}+\bar{x}_{2}^{2}+\bar{p}_{2}^{2}}{2}\right) .
$$

Очевидно, что параметр $I$ является переменной действия на траектории $\Lambda^{1}(I)$ :

$$
I=\frac{1}{2 \pi} \oint_{\Lambda^{1}(E)}\langle p, d q\rangle=\frac{1}{2 \pi} \int_{0}^{2 \pi} p_{\varphi} d \varphi .
$$

Из формул, приведенных в [10], следует, что индекс $\sigma$ комплексного ростка $r^{3}(I)$ на $\Lambda^{1}(I)$ равен нулю. В силу (9) условие квантования параметра $I$ принимает вид $I^{\nu_{1}}(\hbar)=\hbar \nu_{1}$. Тогда формула (10) дает серию квазиклассических уровней энергии, отвечающих семейству орбитально устойчивых боровских фазовых траекторий $\Lambda^{1}(I)(16)$ в атоме водорода:

$$
\begin{aligned}
& E_{\nu}(\hbar)=-\frac{m e_{0}^{4}}{2 \hbar^{2} \nu_{1}^{2}}+\frac{m e_{0}^{4}}{\hbar^{2}\left|\nu_{1}\right|^{3}}\left(\nu_{2}+\nu_{3}+1\right), \\
& \left|\nu_{1}\right| \gg 1, \quad \nu_{1}=0, \pm 1, \pm 2, \ldots, \quad \nu_{2}, \nu_{3}=0,1,2, \ldots .
\end{aligned}
$$

2) В физической литературе $H_{2}(I, \bar{y})$ - гамильтониан исходной задачи в окрестности равновесной окружности $S^{1}(E)$ в приближении малых колебаний. 
В области квантовых чисел $\left|\nu_{1}\right| \gg 1, \nu_{j} /\left|\nu_{1}\right|<1, j=2,3$, формула для $E_{\nu}(\hbar)$ при $\left|\nu_{1}\right| \rightarrow \infty$ асимптотически эквивалентна следующей:

$$
E_{\nu}(\hbar)=-\frac{m e_{0}^{4}}{2 \hbar^{2}} \frac{1}{\left(\left|\nu_{1}\right|+\nu_{2}+\nu_{3}+1\right)^{2}}+O\left(\left(\frac{\nu_{2}+\nu_{3}}{\left|\nu_{1}\right|}\right)^{2}\right) .
$$

Таким образом, "неполномерная" теория (комплексный метод ВКБ) квазиклассического квантования семейства боровских траекторий $\Lambda^{1}(I)(16)$ воспроизводит в главном члене асимптотики по $\hbar \rightarrow 0\left(\nu_{1} \sim 1 / \hbar\right)$ точный спектр атома водорода [37]

$$
E_{n}=-\frac{m e_{0}^{4}}{2 \hbar^{2} n^{2}}, \quad n=1,2, \ldots
$$

для возбужденных уровней $(n \gg 1)$, если считать, что главное квантовое число $n=\left|\nu_{1}\right|+\nu_{2}+\nu_{3}+1$. Подчеркнем при этом, что поиск требуемых для квантования геометрических объектов (траекторий $\Lambda^{1}(I)(16)$ с комплексным ростком $r^{3}(I)$ ) не аппелирует к тому факту, что соответствующая гамильтонова система является интегрируемой.

Боровская фазовая траектория (для интегрируемой системы (13)) является резонансной, и, как мы говорили, это приводит к "бесконечнократному" асимптотическому вырождению квазиклассических уровней энергии $E_{\nu}(\hbar)(19)$. Приведем (см., например, [9]) соответствующие квазиклассические собственные функции $\Psi_{\nu}(q)$ (когерентные состояния), зависящие от двух комплексных параметров $\gamma_{j} \in \mathbb{C}, \operatorname{Re} \gamma_{j}>0$, $j=2,3$ :

$$
\begin{aligned}
\Psi_{\nu}(q)= & \Psi_{\nu}^{\gamma}(q, \hbar)=N(\gamma) e^{i \nu_{1} \varphi} \exp \left(-\omega_{0}^{2} \frac{\gamma_{2}(\rho-R)^{2}}{2 \hbar}\right) \times \\
& \times H_{\nu_{2}}\left(\omega_{0} \sqrt{\gamma_{2}} \frac{\rho-R}{\sqrt{\hbar}}\right) \exp \left(-\omega_{0}^{2} \frac{\gamma_{3} z^{2}}{2 \hbar}\right) H_{\nu_{3}}\left(\omega_{0} \sqrt{\gamma_{3}} \frac{z}{\sqrt{\hbar}}\right),
\end{aligned}
$$

где $R=R\left(\hbar \nu_{1}\right)=\hbar^{2} \nu_{1}^{2} /\left(m e_{0}^{2}\right)$ - квантованный радиус, $\omega_{0}=m e_{0}^{4} /\left(\left|\nu_{1}\right|^{3} \hbar^{3}\right), H_{\nu}-$ полином Эрмита, $N=\omega_{0} \sqrt{\left|\gamma_{2}\right|\left|\gamma_{3}\right|}\left(\pi \sqrt{\hbar 2^{1+\nu_{2}+\nu_{3}} \nu_{2} ! \nu_{3} !}\right)^{-1 / 2}-$ нормировочная константа.

Теперь напомним, как эта же формула (21) возникает в “полномерной” теории квазиклассического квантования трехпараметрических семейств трехмерных лагранжевых торов $\Lambda^{3}$, которые инвариантны относительно интегрируемой гамильтоновой системы с гамильтонианом $H(14)$.

Эти торы определяются в силу теоремы Лиувилля первыми интегралами $F_{1}, F_{2}$, $F_{3}$ системы, попарно находящимися в инволюции. Для атома водорода положим, например, $F_{1}(p, q)=H(p, q), F_{2}=L(p, q)=|\vec{L}|$, где $\vec{L}=p \times q-$ орбитальный момент электрона, и $F_{3}=L_{z}(p, q)$ - проекция момента на ось $z$. В открытой области $\Omega^{6}$ фазового пространства $\mathbb{R}_{p, q}^{6}$, задаваемой как

$$
\Omega^{6}=\left\{(p, q), H(p, q)<0,0<\left|L_{z}(p, q)\right|<L(p, q)\right\},
$$

уравнения $H(p, q)=E, L(p, q)=L_{0}, L_{z}(p, q)=M_{0}$ определяют трехмерные лагранжевы торы $\Lambda^{3}\left(E, L_{0}, M_{0}\right)$, если числа $E, L_{0}, M_{0}$ таковы, что

$$
-\frac{m e_{0}^{4}}{2 L_{0}^{2}}<E<0, \quad 0<\left|M_{0}\right|<L_{0}
$$


В области $\Omega^{6}$ удобно ввести следующие "сферические" канонические координаты: $(r \geqslant 0, \varphi(\bmod 2 \pi), \theta \in[0, \pi))$ - сферические координаты на $\mathbb{R}_{q}^{3}$ и $\left(p_{r}, p_{\varphi}, p_{\theta}\right)$ - канонически сопряженные им импульсы. Тогда тор $\Lambda^{3}\left(E, L_{0}, M_{0}\right)$ является прямым произведением трех связных замкнутых кривых $\Gamma_{1}, \Gamma_{2}, \Gamma_{3}$, где

$$
\begin{array}{ll}
\Gamma_{1}: & L_{z}(p, q)=M_{0} \Longleftrightarrow p_{\varphi}=M_{0}, \quad \varphi(\bmod 2 \pi), \\
\Gamma_{2}: & L^{2}=L_{0}^{2} \Longleftrightarrow p_{\theta}^{2}+\frac{M_{0}^{2}}{\sin ^{2} \theta}=L_{0}^{2}, \\
\Gamma_{3}: & H\left(p_{r}, r, M_{0}, L_{0}\right)=E \Longleftrightarrow \frac{1}{2 m}\left(p_{r}^{2}+\frac{L_{0}^{2}}{r^{2}}\right)-\frac{Z e_{0}^{2}}{r}=E .
\end{array}
$$

Пусть $\left(\varphi_{1}, \varphi_{2}, \varphi_{3}\right)$ - "полярные" угловые переменные вдоль базисных циклов $\Gamma_{j}$, $j=1,2,3$, на торе $\Lambda^{3}\left(E, M_{0}, L_{0}\right)$, причем $\varphi_{3}=\varphi$. Тогда сопряженные им переменные действия $I=\left(I_{1}, I_{2}, I_{3}\right), I_{j}=I_{j}\left(E, M_{0}, L_{0}\right)=(2 \pi)^{-1} \oint_{\Gamma_{j}}\langle p, d q\rangle$, выражаются через значения первых интегралов $\left(E, M_{0}, L_{0}\right) \in \widetilde{\Omega}^{3}$, где $\widetilde{\Omega}^{3}$ задается системой неравенств (22), по формулам [5]

$$
I_{1}=p_{\varphi}=M_{0}, \quad I_{2}=\left(L_{0}-\left|I_{1}\right|\right), \quad I_{3}=\frac{m e_{0}^{2}}{\sqrt{-2 m E}}-L_{0} .
$$

Следовательно, в области $\Omega^{6} \subset \mathbb{R}_{p, q}^{6}$ функция Гамильтона (13) в переменных действие-угол $(I, \varphi)$ имеет вид

$$
H=H(p, q)=H(I)=-\frac{m e_{0}^{4}}{2\left(\left|I_{1}\right|+I_{2}+I_{3}\right)^{2}},
$$

где в силу $(22)$ и $(23)$ переменные действия пробегают открытую область $M_{I} \subset \mathbb{R}_{I}^{3}$ :

$$
I \in M_{I}=\left\{I_{1} \neq 0, I_{2}>0, I_{3}>0\right\} .
$$

Условие квантования Бора-Зоммерфельда-Маслова семейства лагранжевых торов $\Lambda^{3}\left(E, M_{0}, L_{0}\right)$ состоит в выборе при каждом фиксированном $\hbar, \hbar \rightarrow 0$, дискретного набора значений $I^{\nu}(\hbar) \in M_{I}$ в соответствии с формулами (ср. с (12))

$$
I_{j}^{\nu}(\hbar)=\left(\nu_{j}+\frac{M_{j}}{4}\right) \hbar, \quad \nu_{1} \in \mathbb{Z}, \quad \nu_{2}, \nu_{3} \in \mathbb{Z}^{+}, \quad\left|\nu_{j}\right| \sim \frac{1}{\hbar}, \quad j=1,2,3,
$$

где $M_{j}$ - индекс Маслова цикла $\Gamma_{j}$ (см. [10]), $M_{1}=0, M_{2}=M_{3}=2$.

Квантованные значения переменных действия $I_{j}^{\nu}(\hbar)$ определяют с точностью $O\left(\hbar^{2}\right)$ серию квазиклассических уровней энергии

$$
E_{\nu}(\hbar)=H\left(I^{\nu}(\hbar)\right)=-\frac{m e_{0}^{4}}{2 \hbar^{2}\left(\left|\nu_{1}\right|+\nu_{2}+\nu_{3}+1\right)^{2}} .
$$

ЗАМЕчАНИЕ 4. Если в соответствии с классическими обозначениями квантовой физики ввести три целых квантовых числа $m, l, n$ (магнитное, орбитальное и главное, соответственно, [37]), связав их с числами $\nu_{1}, \nu_{2}, \nu_{3}$ соотношениями $\nu_{1}=m$, $\nu_{2}=l-|m|, \nu_{3}+1=n-l$, то из (23)-(25) мы найдем квантованные значения первых 
интегралов системы $M_{0}=m \hbar, L_{0}=(l+1 / 2) \hbar$, где $|m| \leqslant l<n$. При этом квантованное значение энергии (26) дает, так же как и "неполномерная" асимптотика спектра (20), точный спектр атома водорода.

Рассмотрим теперь "граничные" торы, отвечающие особому множеству $\Sigma$ уровней интегралов системы, в котором нарушаются условия теоремы Лиувилля (условия независимости первых интегралов). В трехмерном пространстве параметров $\left(E, M_{0}, L_{0}\right)$ множество $\Sigma$ есть граница области $\widetilde{\Omega}^{3}(22)$, состоящая из цилиндра $\Sigma_{1}=\left\{E=-\frac{m e_{0}^{4}}{2 L_{0}^{2}}\right\}$ и двух плоскостей $\Sigma_{2}=\left\{\left|M_{0}\right|=L_{0}\right\}$. В силу формул $(23)$, (24) на множестве $\Sigma$ некоторые из переменных действия $I$ вырождаются, принимая граничные нулевые значения: $\left.I_{2}\right|_{\Sigma_{2}}=0$ и $\left.I_{3}\right|_{\Sigma_{1}}=0$. При $I_{2} \rightarrow 0$ или $I_{3} \rightarrow 0$ соответствующие базисные циклы $\Gamma_{2}$ и $\Gamma_{3}$ тора $\Lambda^{3}\left(I_{1}, I_{2}, I_{3}\right)$ стягиваются в точку. В результате трехмерный лиувиллев тор гладким образом переходит в изотропный тор меньшей размерности $k, 1 \leqslant k<3$. В частности, однопараметрическое семейство боровских траекторий $\Lambda^{1}\left(I_{1}\right)(16)$ отвечает двум граничным значениям $I_{2}=0$ и $I_{3}=0$, что соответствует значениям первых интегралов системы в точках $\left(E, M_{0}, L_{0}\right) \in l$, где кривая $l$ задается как

$$
l=\Sigma_{1} \cap \Sigma_{2}=\left\{E=-\frac{m e_{0}^{4}}{2 I_{1}^{2}}, L_{0}=\left|I_{1}\right|, M_{0}=I_{1}\right\} .
$$

В соответствии с этим экстраполируем "полномерную” асимптотику спектра в область малых квантовых чисел $\nu_{2}$ и $\nu_{3}$, считая, что $\nu_{2} /\left|\nu_{1}\right| \ll 1, \nu_{3} /\left|\nu_{1}\right| \ll 1,\left|\nu_{1}\right| \gg 1$. Тогда разложение правой части формулы (26) в ряд Тейлора с точностью $O\left(\hbar^{2}\right)$, где $\hbar \sim 1 /\left|\nu_{1}\right|$, дает серию квазиклассических уровней энергии (19), ранее найденную на основе “неполномерной” теории квазиклассического квантования замкнутых траекторий с комплексным ростком.

Очевидно, что при $I_{2}=0\left(I_{3}=0\right)$ лагранжев тор $\Lambda^{3}\left(I_{1}, I_{2}, I_{3}\right)$ вырождается в двумерный изотропный тор $\Lambda_{+}^{2}\left(I_{1}, I_{3}\right)\left(\Lambda_{-}^{2}\left(I_{1}, I_{2}\right)\right)$. Проекция тора $\Lambda_{+}^{2}\left(I_{1}, I_{3}\right)$ на конфигурационное пространство $\mathbb{R}_{q}^{3}$ есть круговое кольцо $D^{2}\left(E, M_{0}\right)$ в плоскости $z=0$ с радиусами окружностей $R_{1}\left(E, M_{0}\right)<R_{2}\left(E, M_{0}\right)$, где $R_{1}, R_{2}$ - радиальные точки поворота эффективного потенциала $V_{\mathrm{eff}}^{L_{0}}=-\frac{Z e_{0}^{2}}{r}+\frac{M_{0}^{2}}{2 m r^{2}}$. Проекция на $\mathbb{R}_{q}^{3}$ траектории движения электрона на торе $\Lambda_{+}^{2}\left(I_{1}, I_{3}\right)$ - это эллипс, лежащий в плоскости $D^{2}\left(E, M_{0}\right)$. Движению на торе $\Lambda_{-}^{2}\left(I_{1}, I_{2}\right)$ отвечает в конфигурационном пространстве движение электрона по окружности радиуса $R\left(E, L_{0}\right)\left(R\left(E, L_{0}\right)\right.$ - корень кубического уравнения $V_{\text {eff }}^{L_{0}}=E$ ), лежащей в плоскости, которая проходит через начало координат и составляет угол $\theta_{0}=\arccos \frac{\left|M_{0}\right|}{L_{0}}$ с плоскостью $z=0$. Подчеркнем, что для гладкого трехпараметрического семейства лиувиллевых торов $\Lambda^{3}(I), I \in \widetilde{\Omega}^{3}$, "граничные" изотропные торы $\Lambda_{ \pm}^{2}$ не изолированны, а также образуют двухпараметрическое семейство.

Ясно, что помимо этих торов для системы с гамильтонианом (13), в которой при $E<0$ все фазовые траектории замкнуты, существуют и другие двумерные торы, которые можно получать объединением этих траекторий. Если в результате такого

4 Теоретическая и математическая физика, т. 151, № 2, 2007 г. 
объединения возникает изотропный тор, то он может служить геометрическим объектом, пригодным для квантования комплексным методом ВКБ. Важным и естественным примером таких торов как для атома водорода, так и для атома гелия являются двумерные изотропные торы $\Lambda^{2}$, полученные "объединением" боровских фазовых траекторий $\Lambda^{1}(E)(16)$ с помощью сдвигов вдоль $2 \pi$-периодических траекторий гамильтониана $L_{x}$ или $L_{y}$. Например, если через $\tau_{x}$ обозначить “время" вдоль траекторий системы Гамильтона с гамильтонианом $L_{x}$ и через $g^{\tau_{x}}-$ фазовый поток, отвечающий $L_{x}$, то очевидно, что $\Lambda_{x}^{2}=g^{\tau_{x}} \Lambda^{1}\left(I_{1}\right)$ есть двумерный тор с координатами $\tau_{x}(\bmod 2 \pi)$ и $\varphi(\bmod 2 \pi)$, где $\varphi-$ угловая переменная на кривой $\Lambda^{1}$, причем этот тор является изотропным (гамильтониан $L_{x}$ есть симметрия $H$ ). Интересно отметить, что проекция тора $\Lambda_{x}^{2}$ на $\mathbb{R}_{x}^{3}$ есть двумерная сфера $S^{2}(E)$ (боровская орбита $S^{1}(E)$ на рис. $1 б$ является для нее экватором). Полюсы этой сферы суть особые (фокальные) точки отображения проекции $\pi_{x}: \Lambda_{x}^{2} \rightarrow \mathbb{R}_{q}^{3}$, в которых ранг отображения $d \pi_{x}$ падает на единицу.

Отметим, что в отличие от "граничных" торов $\Lambda_{ \pm}^{2}$ семейства $\Lambda^{3}\left(I_{1}, I_{2}, I_{3}\right)$ эти двумерные торы $\left(\Lambda_{x}^{2}, \Lambda_{y}^{2}\right)$ образуют лишь однопараметрическое семейство $\Lambda_{x, y}^{2}=$ $\Lambda_{x, y}^{2}\left(I_{1}\right)$. Поэтому непосредственно формулы квантования (11) для этого семейства применить нельзя, хотя бы потому, что число условий квантования больше числа свободных параметров, подлежащих квантованию. В этой ситуации, когда число $l$ переменных действия $I=\left(I_{1}, \ldots, I_{l}\right)$ на торе $\Lambda^{k}$ меньше, чем $k$, соответствующая модификация теории, использующая обобщенные переменные действие-угол и переменные гармонического осциллятора в полной окрестности устойчивого изотропного тора [18], дана в [10]. Примеры однопараметрических семейств двумерных изотропных торов для гамильтониана атома гелия мы приведем ниже в разделе 3.

Сформулируем теперь основной результат этой работы.

Теорема. Квазиклассическая спектральная серия, отвечающая в пределе $\hbar \rightarrow 0$ семейству боровских орбит $S^{1}(E)$ (рис. 1б) в атоме гелия, имеет следующий вид.

Квазиклассические уровни энергии (в атомной системе единии) суть

$$
\begin{aligned}
& E_{l, \nu}=E_{l, \nu_{1}, \nu_{2}, \nu_{3}, \nu_{4}}=-\frac{4(4 Z-1)^{2}}{\left(l+1 / 2+\sum_{j=2}^{4} B_{j}(Z)\left(\nu_{j}+1 / 2\right)\right)^{2}}, \\
& \nu_{j}=0,1,2, \ldots, \quad l=2,3,4 \ldots, \quad l \gg \nu_{j}, \quad j=2,3,4,
\end{aligned}
$$

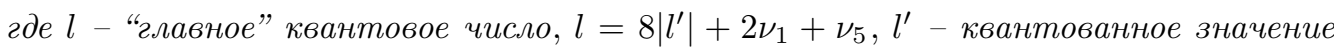
полного орбитального момента $L$ двух электронов на боровской орбите, а числа $\nu_{2}, \nu_{3}, \nu_{4}$ нумеруют моды малых колебаний (радиальных и аксиальных) около равновесной боровской орбиты электронов $S^{1}(E)$ (cм. pис. 1$), \nu_{1}, \nu_{5}=0,1,2, \ldots$, $l^{\prime}=0, \pm 1, \pm 2, \ldots ;$ числа $B_{j}(Z)$ определяются формулами

$$
\begin{aligned}
& B_{2}(Z)=8 \sqrt{\frac{Z}{4(4 Z-1)}}, \quad B_{3}(Z)=4 \sqrt{\frac{4 Z+1}{4 Z-1}}, \\
& B_{4}(Z)=4 \sqrt{\frac{8 Z-1}{2(4 Z-1)}} .
\end{aligned}
$$


Квазиклассические (нормированные) волновые функиии, отвечающие $E_{l, \nu}(27)$, задаются как

$$
\Psi_{l, \nu}^{\gamma} \equiv \Psi_{E_{l, \nu}}^{\gamma}(q)=N_{l^{\prime}, \nu} e^{i l^{\prime} \Phi} \Psi_{\nu_{1}, \nu_{2}}\left(z_{1}, z_{2}, \hbar\right) \Psi_{\nu_{3}, \nu_{4}, \nu_{5}}^{\gamma}\left(\rho_{1}, \rho_{2}, \theta, \hbar\right), \quad \gamma \in \mathbb{C}, \quad \operatorname{Im} \gamma>0
$$

$$
\begin{gathered}
\Psi_{\nu_{1}, \nu_{2}}\left(z_{1}, z_{2}, \hbar\right)=e^{\frac{i\left\langle B C^{-1} \vec{\mu}, \vec{\mu}\right\rangle}{2}} \prod_{j=1}^{2} \Phi_{j}\left(z_{1}, z_{2}, \hbar\right), \\
\Psi_{\nu_{3}, \nu_{4}, \nu_{5}}^{\gamma}\left(\rho_{1}, \rho_{2}, \theta, \hbar\right)=e^{i\left\langle B_{\gamma} C_{\gamma} \vec{\eta}, \vec{\eta}\right\rangle / 2} \prod_{k=3}^{5} \Phi_{k}^{\gamma}\left(\rho_{1}, \rho_{2}, \theta, \hbar\right) .
\end{gathered}
$$

Здесъ $\rho_{i}, \varphi_{i}, z_{i}-$ полярные координаты $i$-го электрона; $\Phi=\varphi_{1}+\varphi_{2}, \theta=\varphi_{1}-$ $\varphi_{2}, \omega_{0}=\sqrt{\frac{(4 Z-1) e_{0}^{2}}{m R^{3}}}-$ частота вращения электронов на квантованной боровской орбите радиуса $R=R\left(L_{z}=l^{\prime} \hbar\right)=\frac{4 \hbar^{2} l^{\prime 2}}{m e_{0}^{2}(4 Z-1)}, \vec{\eta}=\hbar^{-1 / 2}\left(\rho_{1}-R, \rho_{2}-R, \theta \pi\right), \mu=$ $\hbar^{-1 / 2}\left(z_{1}, z_{2}\right) ; \Phi_{j}^{\gamma}\left(z_{1}, z_{2}, \hbar\right)=H_{\nu_{j}}\left(\left\langle\vec{\mu}, \vec{A}_{j}\right\rangle\right), j=1,2, u \Phi^{\gamma}\left(\rho_{1}, \rho_{2}, \theta, \hbar\right)=H_{\nu_{k}}\left(\left\langle\vec{\eta}, \vec{A}_{k}^{\gamma}\right\rangle\right)$, $k=3,4,5, H_{\nu}(\cdot)$ - многомерные полиномь Эрмита; $\vec{A}_{j}-j$-й столбец матриць $C^{-1}, \vec{A}_{i+2}^{\gamma}-i$-й столбеи, матрицъ $C_{\gamma}^{-1}$. Матрицы $B, C$ и $B_{\gamma}, C_{\gamma}$ определяются формулами

$$
\begin{aligned}
& B=i \sqrt{\frac{m \omega_{0}}{2}}\left(\begin{array}{cc}
1 & d \\
-1 & d
\end{array}\right), \quad C=\frac{1}{\sqrt{2 m \omega_{0}}}\left(\begin{array}{cc}
1 & 1 / d \\
-1 & 1 / d
\end{array}\right), \quad d=\left(\frac{4 Z}{4 Z-1}\right)^{1 / 4}, \\
& B_{\gamma}=i \sqrt{2 m \omega_{0}}\left(\begin{array}{ccc}
b_{1} & b_{2} / \sqrt{5} & b_{3}(1-\gamma) \\
b_{1} & 2 b_{2} / \sqrt{5} & b_{3}(1-\gamma) \\
0 & 0 & -b_{3}(1-\gamma)
\end{array}\right) \\
& b_{1}=\left(\frac{12 Z}{4 Z-1}\right)^{1 / 4}, \quad b_{2}=\left(\frac{24 Z+3}{8 Z-2}\right)^{1 / 4} \text {, } \\
& b_{3}=\frac{1}{2}\left(\frac{8}{4 Z-1}+\frac{1}{128}\right)^{1 / 4}\left(\frac{64 Z^{2}-196}{4 Z-1}\right)^{1 / 2} \text {, } \\
& C_{\gamma}=i \sqrt{2 m \omega_{0}}\left(\begin{array}{ccc}
b_{1}^{-1} & \frac{b_{2}^{-1}}{\sqrt{5}} & c_{1}(1+\gamma) \\
b_{1}^{-1} & \frac{2 b_{2}^{-1}}{\sqrt{5}} & c_{1}(1+\gamma) \\
b_{1}^{-1} \frac{6 Z-1}{64 Z^{2}-196} & \frac{3 b_{2}^{-1}}{\sqrt{20}} \frac{128 Z^{2}+4 Z-393}{32 Z^{2}-98} & -c_{2}(1+\gamma)
\end{array}\right) \text {, } \\
& c_{1}=\frac{64 Z^{2}+4 Z-197}{64 Z^{2}-98} \frac{c_{2}}{2}, \quad c_{2}=\sqrt{\frac{64 Z^{2}-196}{4 Z-1}}\left(\frac{8}{4 Z-1}+\frac{1}{128}\right)^{-1 / 4},
\end{aligned}
$$

причем $|\gamma|<1, N_{l^{\prime}, \nu}-$ нормировочная постоянная.

\section{2. БОРОВСКАЯ ФАЗОВАЯ ТРАЕКТОРИЯ С КОМПЛЕКСНЫМ РОСТКОМ}

В ограниченной заряженной задаче трех тел гамильтонова система с гамильтонианом (1) является неинтегрируемой по Лиувиллю, однако допускает группы 
симметрий, в частности, непрерывную группу трехмерных вращений $G$ фазового пространства, соответствующую группе вращений $S O_{3} \times S O_{3}$ в $\mathbb{R}_{q_{1}, q_{2}}^{6}$, относительно которой потенциал $V\left(q_{1}, q_{2}\right)$ инвариантен. Тогда, как известно [6], пуассоново действие группы $G$ на $T^{*}\left(\mathbb{R}_{q}^{6}\right)=\mathbb{R}_{p, q}^{12}$ порождает отображение момента, компонентами которого являются проекции $L_{1}, L_{2}, L_{3}$ (коммутирующие с гамильтонианом $H$ : $\left.\left\{H, L_{j}\right\}=0, j=1,2,3\right)$ полного орбитального момента $L=\left(L_{1}, L_{2}, L_{3}\right)$ двух электронов, $L=q_{1} \times p_{1}+q_{2} \times p_{2}$.

Неинтегрируемый гамильтониан $H(1)$ обладает группой симметрий, что позволяет провести редукцию исходной системы к системе меньшей размерности и тем самым упростить задачу поиска инвариантных изотропных многообразий $\Lambda^{k}$, пригодных для квазиклассического квантования методом комплексного ростка.

Простейшим примером, иллюстрирующим возможности такой редукции, являются боровские орбиты, представленные на рис. 1б. Траектории коррелированного движения двух электронов вдоль окружности $S_{R}$ радиуса $R$ представляет собой проекцию замкнутой фазовой траектории $\Lambda^{1}(E)$ на физический аналог конфигурационного пространства $\mathbb{R}_{x, y, z}^{3}$. Эти траектории суть ОПР (стационарные вращения) исходной гамильтоновой системы

$$
\dot{p}=-H_{q}, \quad \dot{q}=H_{p}, \quad(p, q) \in \mathbb{R}^{12} .
$$

ОПР определяются равновесными состояниями (точками покоя) редуцированного (приведенного) гамильтониана на приведенном фазовом пространстве, которое представляет собой факторизацию исходного фазового пространства по уровням проекции полного момента на ось $z: L_{3}(p, q)=L_{z}, L_{z}=$ const $>0$.

Для перехода к приведенному фазовому пространству $M_{L_{z}} \sim \mathbb{R}^{10}$ и редуцированному гамильтониану $H_{L_{z}}$ в нашем случае достаточно исключить циклическую переменную, что удобнее проделать в полярных координатах на конфигурационном пространстве $\mathbb{R}^{6}=\mathbb{R}_{q_{1}}^{3} \times \mathbb{R}_{q_{2}}^{3}: q^{\prime}=\left(\rho_{1}, \rho_{2}, z_{1}, z_{2}, \varphi_{1}, \varphi_{2}\right), \varphi_{j} \in[0,2 \pi], \rho_{j} \geqslant 0, j=1,2$.

В координатах $q^{\prime}$ и $p^{\prime}$, где $p^{\prime}=\left(p_{\rho_{1}}, p_{\rho_{2}}, p_{z_{1}}, p_{z_{2}}, p_{\varphi_{1}}, p_{\varphi_{2}}\right)$ - сопряженные импульсы, координата $\phi=\varphi_{1}+\varphi_{2}(\bmod 2 \pi)$ в гамильтониане

$$
\begin{aligned}
H\left(p^{\prime}, q^{\prime}\right)= & \frac{1}{2 m} \sum_{j=1}^{2}\left(p_{\rho_{j}}^{2}+\frac{1}{\rho_{j}^{2}} p_{\varphi_{j}}^{2}+p_{z_{j}}^{2}\right)- \\
& \quad-\sum_{j=1}^{2} \frac{Z e_{0}^{2}}{\sqrt{\rho_{j}^{2}+z_{j}^{2}}}+\frac{e_{0}^{2}}{\sqrt{\rho_{1}^{2}+\rho_{2}^{2}-2 \rho_{1} \rho_{2} \cos \left(\varphi_{1}-\varphi_{2}\right)+\left(z_{1}-z_{2}\right)^{2}}}
\end{aligned}
$$

очевидно, является циклической, $p_{\Phi}=p_{\varphi_{1}}+p_{\varphi_{2}}=$ const $=L_{z}$ - момент, сопряженный этой координате. Тогда на редуцированном фазовом пространстве с координатами $(\tilde{p}, \tilde{q})=\left(p_{\rho_{1}}, p_{\rho_{2}}, p_{z_{1}}, p_{z_{2}}, p_{\theta}, \rho_{1}, \rho_{2}, z_{1}, z_{2}, \theta\right)$, где $\theta=\varphi_{1}-\varphi_{2}$, редуцированный гамильтониан $H_{L_{z}}(\tilde{q}, \tilde{p})$, зависящий от интеграла $L_{z}$ как от параметра, принимает 

вид

$$
\begin{gathered}
H_{L_{z}}(\tilde{q}, \tilde{p})=\sum_{j=1}^{2}\left(\frac{1}{2 m}\left(p_{\rho_{j}}^{2}+p_{z_{j}}^{2}\right)-\frac{Z e_{0}^{2}}{\sqrt{\rho_{j}^{2}+z_{j}^{2}}}\right)+\frac{1}{2 m \rho_{1}^{2}}\left(p_{\theta}+L_{z}\right)^{2}+\frac{1}{2 m \rho_{2}^{2}}\left(L_{z}-p_{\theta}\right)^{2}+ \\
+\frac{e_{0}^{2}}{\sqrt{\rho_{1}^{2}+\rho_{2}^{2}-2 \rho_{1} \rho_{2} \cos \theta+\left(z_{1}-z_{2}\right)^{2}}}
\end{gathered}
$$

Точки покоя $r^{*}\left(L_{z}\right)=\left(\tilde{p}_{*}, \tilde{q}_{*}\right)$ редуцированного гамильтониана $H_{L_{z}}$ на $\mathbb{R}_{\tilde{p}, \tilde{q}}^{10}$ определяют семейство ОПР исходной системы (замкнутых фазовых кривых $\Lambda^{1}(E)$ ), где $E-$ классическая энергия, $E<0$.

УтВеРЖДЕНИЕ. Для боровских орбит $S^{1}(E)$ (рис. 1б) в гелиеподобном атоме соответствующее семейство ОПР (боровских фазовых траекторий $\left.\Lambda^{1}(E)\right)$ задается формулами

$$
\Lambda^{1}(E)=\left(\Phi=\Phi(t)=\omega_{0}\left(L_{z}\right) t+\Phi_{0}, p_{\Phi}=L_{z}\right) \times\left. r^{*}\left(L_{z}\right)\right|_{L_{z}=f^{-1}(E)},
$$

əəe

$$
\begin{gathered}
r^{*}\left(L_{z}\right)=\left(p_{\rho_{1}}=0, p_{\rho_{2}}=0, p_{z_{1}}=0, p_{z_{2}}=0, p_{\theta}=0,\right. \\
\left.\rho_{1}=R\left(L_{z}\right), \rho_{2}=R\left(L_{z}\right), z_{1}=0, z_{2}=0, \theta=\pi\right), \\
R=R\left(L_{z}\right)=\frac{L_{z}^{2}}{m e_{0}^{2}(4 Z-1)}, \quad \omega_{0}\left(L_{z}\right)=\frac{2\left|L_{z}\right|}{m R^{2}\left(L_{z}\right)},
\end{gathered}
$$

$u$

$$
\left.H\right|_{\Lambda^{1}(E)}=E=f\left(R\left(L_{z}\right)\right)=-\frac{(4 Z-1) e_{0}^{2}}{4 R\left(L_{z}\right)} .
$$

ДоКАЗАТЕЛЬСтво следует из явного вида редуцированного гамильтониана (33) и проводится прямым дифференцированием.

Критическая точка $r^{*}\left(L_{z}\right)$ приведенного гамильтониана невырождена и устойчива в линейном приближении: все собственые значения $\lambda_{j}^{ \pm}, j=1,2, \ldots, 5$, постоянной $(10 \times 10)$-матрицы $A\left(L_{z}\right)=J d^{2} H_{L_{z}}\left(r^{*}\left(L_{z}\right)\right)$ лежат на мнимой оси (равномерно по $L_{z}$, $\left.\left|L_{z}\right|>0, Z \geqslant 2\right), \lambda_{j}^{ \pm}= \pm i \omega_{j}\left(L_{z}\right)$, где $\omega_{j}\left(L_{z}\right)>0$ - нормальные частоты малых колебаний вблизи положения равновесия $r^{*}\left(L_{z}\right)$. Имеет место следующее утверждение.

Лемма. Собственные значения $\lambda_{j}^{ \pm}$и соответствующие собственные векторстолбиљ $f_{j}^{ \pm}=\left(w_{j}^{ \pm}, z_{j}^{ \pm}\right), j=1, \ldots, 5$, матрицы $A\left(L_{z}\right)$, нормированные условием

$$
\left\langle f_{j}^{ \pm}, J \bar{f}_{j}^{ \pm}\right\rangle= \pm 2 i,
$$


где черта над вектором означает комплексное сопряжение, задаются соотношениями

$$
\begin{aligned}
& \lambda_{j}^{ \pm}= \pm i \omega_{j}\left(L_{z}\right), \quad \omega_{1}=\sqrt{\frac{(4 Z-1) e_{0}^{2}}{4 R^{3}\left(L_{z}\right) m}}, \\
& \omega_{2}=\sqrt{\frac{Z e_{0}^{2}}{R^{3}\left(L_{z}\right) m}}, \quad \omega_{3}=\sqrt{\frac{(16 Z+4) e_{0}^{2}}{16 R^{3}\left(L_{z}\right) m}}, \\
& \omega_{4}=\sqrt{\frac{(8 Z-1) e_{0}^{2}}{8 R^{3}\left(L_{z}\right) m}}, \quad \omega_{5}=\sqrt{\frac{(4 Z-1) e_{0}^{2}}{64 R^{3}\left(L_{z}\right) m}} ; \\
& f_{1}^{ \pm}=\left( \pm-i m \omega_{1}, \mp i m \omega_{1}, 0,0,0,1,-1,0,0,0\right)^{\mathrm{T}} N_{1} \text {, } \\
& f_{2}^{ \pm}=\left( \pm i m \omega_{2}, \pm i m \omega_{2}, 0,0,0,1,1,0,0,0\right)^{\mathrm{T}} N_{2}, \\
& f_{3}^{ \pm}=\left(0,0, \pm i m \sqrt{\omega_{3}^{2}+2 \omega_{4}^{2}}, \pm i m \sqrt{\omega_{3}^{2}+2 \omega_{4}^{2}}, 0,0,0, \frac{1}{2}, \frac{1}{2}, \frac{6 Z-1}{64 Z^{2}-196}\right)^{\mathrm{T}} N_{3}, \\
& f_{4}^{ \pm}=\left(0,0, \pm \frac{2 i}{3} m \sqrt{2 \omega_{3}^{2}+\omega_{4}^{2}}, \pm \frac{4 i}{3} m \sqrt{2 \omega_{3}^{2}+\omega_{4}^{2}}, 0,0,0, \frac{2}{3}, \frac{4}{3}, \frac{128 Z^{2}+4 Z-393}{32 Z^{2}-98}\right)^{\mathrm{T}} N_{4}, \\
& f_{5}^{ \pm}=\left(0,0, \pm \frac{i}{2} m \sqrt{\omega_{3}^{2}+\omega_{4}^{2}+8 \omega_{5}^{2}}, \pm \frac{i}{2} m \sqrt{\omega_{3}^{2}+\omega_{4}^{2}+8 \omega_{5}^{2}}, \mp \frac{i}{2} m \sqrt{\omega_{3}^{2}+\omega_{4}^{2}+8 \omega_{5}^{2}}, 0,0,\right. \\
& \left.\frac{1}{2} \frac{64 Z^{2}+4 Z-197}{64 Z^{2}-196}, \frac{1}{2} \frac{64 Z^{2}+4 Z-197}{64 Z^{2}-196}, 1\right)^{\mathrm{T}} N_{5},
\end{aligned}
$$

где нормировочные постоянные суть

$$
\begin{aligned}
& N_{1}=\left(2 m \omega_{1}\right)^{-1 / 2}, \quad N_{2}=\left(2 m \omega_{2}\right)^{-1 / 2}, \quad N_{3}=\left(m \sqrt{\omega_{3}^{2}+2 \omega_{4}^{2}}\right)^{-1 / 2}, \\
& N_{4}=\frac{3}{\sqrt{20}}\left(m \sqrt{2 \omega_{3}^{2}+\omega_{4}^{2}}\right)^{-1 / 2}, \quad N_{5}=\left(\frac{4 Z-1}{64 Z^{2}-196}\right)^{-1 / 2}\left(m \sqrt{\omega_{3}^{2}+\omega_{4}^{2}+8 \omega_{5}^{2}}\right)^{-1 / 2} .
\end{aligned}
$$

ДОКАЗАТЕЛЬСТвО проводится стандартными вычислениями и сводится к получению явных формул для корней кубического уравнения с двумя параметрами $R$ и $Z$.

Из предыдущих рассуждений следует, что приведенный оператор монодромии $e^{A\left(L_{z}\right) t}$ соответствующей системы в вариациях (линеаризации системы (32) на траекториях $\left.\Lambda^{1}(E)(34)\right)$ диагонализуем, и его спектр лежит на единичной окружности. Иными словами, траектория $\Lambda^{1}(E)$ орбитально устойчива (в линейном приближении): все решения линеаризованной системы (в координатах $\left.\left(p^{\prime}, q^{\prime}\right)\right)$, косоортогональные решению $a_{6}=(\dot{P}, \dot{Q})(t)$, ограничены при $t \in \mathbb{R}$. В координатах $\left(p^{\prime}, q^{\prime}\right)$ в $\mathbb{R}^{12}$ траектория $\Lambda^{1}(E)$, очевидно, определяется вектор-функциями

$$
\begin{gathered}
P\left(t, L_{z}\right)=\left(0,0,0,0, L_{z}, L_{z}\right)^{\mathrm{T}}, \quad Q\left(t, L_{z}\right)=(0,0,0,0, \Phi(t), \Phi(t)+\pi)^{\mathrm{T}}, \\
\Phi(t)=\omega_{0}\left(L_{z}\right) t+\Phi_{0}(\bmod 2 \pi) .
\end{gathered}
$$

Определим по собственным векторам $f_{j}^{ \pm}=\left(w_{j}^{ \pm}, z_{j}^{ \pm}\right)^{\mathrm{T}}$ 12-мерные комплексные векторы $a_{j}\left(L_{z}\right)=\left(w_{j}^{+}, 0, z_{j}^{+}, 0\right)^{\mathrm{T}}, j=1,2, \ldots, 5$. Сопоставим теперь каждой точке 
$\phi \in \Lambda^{1}(E)$ линейную однопараметрическую $\left(\gamma \in \mathbb{C}-\right.$ параметр) оболочку $\tilde{r}_{\gamma}^{5}(\phi)$ векторов $a_{j}^{\gamma}\left(\phi, L_{z}\right)$ :

$$
\tilde{r}_{\gamma}^{5}(\phi)=\left\{a \in \mathbb{C}^{12}, a=\sum_{j=1}^{5} \alpha_{j} a_{j}^{\gamma}\left(\phi, L_{z}\right), \alpha_{j} \in \mathbb{C}\right\} .
$$

Здесь

$$
\begin{aligned}
& a_{j}^{\gamma}\left(\phi, L_{z}\right)=e^{i \omega_{j}\left(L_{z}\right) \phi / \omega_{0}\left(L_{z}\right)} a_{j}\left(L_{z}\right), \quad j=1, \ldots, 4, \\
& a_{5}^{\gamma}\left(\phi, L_{z}\right)=\left(1-|\gamma|^{2}\right)^{-1 / 2} e^{i \omega_{5}\left(L_{z}\right) \phi / \omega_{0}\left(L_{z}\right)}\left(a_{5}\left(L_{z}\right)+\gamma \bar{a}_{5}\left(L_{z}\right)\right) .
\end{aligned}
$$

При этом выполнено условие нормировки $\left\{a_{5}^{\gamma}, \bar{a}_{5}^{\gamma}\right\}=2 i, \operatorname{Im} \gamma>0,|\gamma|<1$.

Тогда нетрудно проверить, что комплексная плоскость $r_{\gamma}^{6}(\phi)$, определенная формулой

$$
r_{\gamma}^{6}(\phi)=\tilde{r}_{\gamma}^{5}(\phi) \oplus{ }^{\mathbb{C}} T_{\phi} \Lambda^{1}(E), \quad \phi \in \Lambda^{1}(E),
$$

где ${ }^{\mathbb{C}} T_{\phi} \Lambda^{1}(E)$ - комплексификация касательной прямой к кривой $\Lambda^{1}(E)$ в точке $\phi \in \Lambda^{1}(E)$ (вдоль вектора $a_{6}=(\dot{P}, \dot{Q})$ ), удовлетворяет (с учетом условия нормировки (36)) всем аксиомам комплексного ростка, причем соответствующий комплексный росток $r_{\gamma}^{6}$ на траектории $\Lambda^{1}(E)$ является инвариантным (см. [9]).

Следуя алгоритму квантования инвариантных фазовых траекторий с комплексным ростком [7]-[9], сопоставим семейству боровских орбит с комплексным ростком $\left[\Lambda^{1}(E), r_{\gamma}^{6}\right]$ спектральную серию исходной квантовой задачи $\left(E_{\nu}, \Psi_{\nu}\right)$. Формулы для асимптотических собственных значений определены в (9)-(10), а соответствующие асимптотические собственные функции дает канонический оператор Маслова $\widehat{K}$ на семействе $\left[\Lambda^{1}(E), r_{\gamma}^{6}\right]$ :

$$
\Psi_{\nu}^{\gamma}(q, \hbar)=\left(\widehat{K}_{\left[\Lambda^{1}, r_{\gamma}^{6}\right]} 1\right)(q, \hbar)=\frac{e^{i / \hbar} S^{\gamma}(q)}{\sqrt{\operatorname{det} C(\phi)}} \prod_{j=1}^{5} \Lambda_{j}^{\nu_{j}} 1, \quad q=(\tilde{q}, \phi)
$$

где фаза задается равенством

$$
S^{\gamma}(\tilde{q}, \phi)=\int_{t_{0}}^{t}\langle P, d X\rangle+\langle P(\phi), \tilde{q}-X(\phi)\rangle+\frac{1}{2}\left\langle\tilde{q}-X(\phi), B_{\gamma} C_{\gamma}^{-1}(\phi)(\tilde{q}-X(\phi))\right\rangle,
$$

$B_{\gamma}(\phi)$ и $C_{\gamma}(\phi)-(5 \times 5)$-матрицы, составленные из вектор-столбцов решений системы в вариациях $f_{j}^{+}=\left(w_{j}^{+}, z_{j}^{+}\right)^{\mathrm{T}}, j=1, \ldots, 5$,

$$
\begin{array}{cl}
B_{\gamma}(\phi)=\left(w_{1}, \ldots, w_{4}, w_{5}^{\gamma}\right), & C_{\gamma}(\phi)=\left(z_{1}, \ldots, z_{4}, z_{5}^{\gamma}\right), \\
w_{5}^{\gamma}=\left(1-|\gamma|^{2}\right)^{-1 / 2}\left(w_{5}+\gamma \bar{w}_{5}\right), & z_{5}^{\gamma}=\left(1-|\gamma|^{2}\right)^{-1 / 2}\left(z_{5}+\gamma \bar{z}_{5}\right),
\end{array}
$$

$\Lambda_{j}$ - дифференциальные операторы первого порядка (обобщенные операторы "рождения", см. [7])

$$
\Lambda_{j}=\frac{\sqrt{\hbar}}{i}\left\langle\bar{z}_{j}(\phi), \frac{\partial}{\partial \tilde{q}}\right\rangle-\frac{1}{\sqrt{\hbar}}\left\langle\bar{w}_{j}(\phi)-B_{\gamma} C_{\gamma}^{-1}(\phi) \bar{z}_{j}(\phi), \tilde{q}-X(\phi)\right\rangle .
$$


Подставив в эти формулы результаты утверждения и леммы, после несложных вычислений приходим к функции $\Psi_{\nu}^{\gamma}$ из теоремы.

Таким образом, семейству боровских фазовых траекторий $\Lambda^{1}(E)(34)$ с комплексным ростком $r_{\gamma}^{6}(37)$ отвечает в силу формул (9)-(10) серия квазиклассических $(\hbar \rightarrow 0)$ уровней энергии гелиеподобного атома

$$
E_{l^{\prime}, \nu}(\hbar)=-\frac{(4 Z-1)^{2}}{4 L_{z}^{2}}+\left.\hbar \sum_{j=1}^{5} \omega_{j}\left(L_{z}\right)\left(\nu_{j}+\frac{1}{2}\right)\right|_{L_{z}=l^{\prime} \hbar},
$$

где $L_{z}=l^{\prime} \hbar$ - квантованные значения полного орбитального момента двух электронов на боровской орбите, $l^{\prime} \in \mathbb{Z},\left|l^{\prime}\right| \gg 1$ и $\left|l^{\prime}\right| \hbar \sim 1$ при $\hbar \rightarrow 0, \nu_{j} \in \mathbb{Z}_{+}, j=1,2, \ldots, 5$.

Уровни энергии (38) можно представить (с точностью $O\left(\hbar^{3 / 2}\right)$ ), как и в примере атома водорода (см. раздел 1), в виде ридберговской спектральной серии

$$
\begin{gathered}
E_{l^{\prime}, \nu}(\hbar)=-\frac{(4 Z-1)^{2}}{4 \hbar^{2}\left(\left|l^{\prime}\right|+\sum_{j=1}^{5} \frac{\omega_{j}}{\omega_{0}}\left(l^{\prime} \hbar\right)\left(\nu_{j}+\frac{1}{2}\right)\right)^{2}}, \\
l^{\prime} \in \mathbb{Z}, \quad \nu_{j} \in \mathbb{Z}_{+}, \quad \frac{\nu_{j}}{\left|l^{\prime}\right|} \ll 1 .
\end{gathered}
$$

Отметим, что в силу формул леммы боровская фазовая траектория является резонансной [19]: имеет место резонанс восьмого порядка $8 \omega_{5}=\omega_{0}$ между частотой $\omega_{5}$ фазовых колебаний по угловой переменной $\theta$ в окрестности $\theta=\pi$ и частотой вращения электронов $\omega_{0}$ по равновесной боровской орбите, а также резонанс ворого порядка $2 \omega_{1}=\omega_{0}$ между частотой аксиальных колебаний одного из электронов и частотой $\omega_{0}$. В формуле (39) этот резонанс частот приводит к перенумерации уровней энергии $\left|l^{\prime}\right| \rightarrow l=8\left|l^{\prime}\right|+2 \nu_{1}+\nu_{5}$, так что ридберговская спектральная серия, отвечающая семейству резонансных боровских фазовых траекторий с комплексным ростком, окончательно принимает вид (27), где $B_{j}=8 \omega_{j} / \omega_{0}, j=2,3,4$.

ЗАмЕчАНИЕ 5. С точки зрения геометрических объектов $\left[\Lambda^{1}(E), r_{\gamma}^{6}\right]$, порождающих квазиклассическую спектральную серию соответствующей спектральной квантовой задачи, обнаруженные резонансы приводят к тому, что инвариантный комплексный росток (точнее, его фактор $\tilde{r}^{5}$ ) в каждой точке $\phi \in \Lambda^{1}(E)$ определяется неединственным образом. Существует семейство инвариантных комплексных ростков $\tilde{r}_{\gamma, \delta}^{5}$, зависящее от двух комплексных параметров $\gamma$ и $\delta$. В теореме для простоты мы ограничились случаем, когда $\delta=0$, а $\gamma=\gamma_{1}+i \gamma_{2}, \gamma_{2}>0, \gamma_{1}^{2}+\gamma_{2}^{2}<1\left(\tilde{r}_{\gamma, 0}^{5}=\tilde{r}_{\gamma}^{5}\right)$. Поскольку этот параметр входит в формулы для квазиклассических собственных функций $\Psi_{l, \nu}^{\gamma}(\hbar)(29)$, отвечающих $E_{l, \nu}(\hbar)$, то неединственность комплексного ростка в данном случае приводит, как и в случае атома водорода, к "асимптотически непрерывному" спектру $E_{l, \nu}(\hbar)(27)$.

\section{3. ЗАКЛЮЧЕНИЕ И ОБСУЖДЕНИЕ РЕЗУЛЬТАТОВ}

\section{1. Квазиклассическое квантование устойчивых конфигураций элек-} тронов в атоме гелия. Для атома гелия проблема поиска его классических замкнутых орбит, как и проблема их квантования, имеет длинную историю. Вслед за 
работами [1] еще в 1921 г. Ленгмюр в работе [38] привел результаты численного расчета орбит, отвечающих планарному коррелированному движению двух электронов (в плоскости $z=0$ с общим угловым моментом $\mathbf{L}=0$ ), и получил квазиклассические уровни энергии по формулам “старой” квантовой теории Бора. В настоящее время в физической литературе периодические коррелированные движения электронов в атоме гелия являются предметом интенсивного (в основном численного) исследования (см., например, [39] и цитируемую там литературу). Это связано с тем, что такие типы движения при условии их классической (линейной) стабильности порождают дважды возбужденные (с главными квантовыми числами $n \sim 10$ и выше [40]) долгоживущие состояния квантовой системы несмотря на то, что их энергия выше, чем энергия одноэлектронной ионизации атома [41]. Такие состояния можно наблюдать в экспериментах по мультифотонному лазерному возбуждению гелиеподобных атомов [42], где они проявляются в виде узких резонансов, энергия которых чрезвычайно близка к энергии двойной ионизации атома. Лишь сравнительно недавно в [43] была доказана стабильность ленгмюровских орбит, а также найдена устойчивая конфигурация коррелированного коллинеарного движения электронов (с общим угловым моментом $\mathbf{L}=0$ ), находящихся по одну сторону от ядра, - так называемая $Z^{2+} e^{-} e^{-}$-конфигурация или "frozen planet configuration" [44]. На основе эвристической полуклассической теории Гутцвиллера [45] были вычислены в [44], [46] тройные (с тремя квантовыми числами) ридберговские серии уровней энергии дважды возбужденных состояний, симметричных $\left(\left\langle r_{1}\right\rangle \sim\left\langle r_{2}\right\rangle\right)$ для ленгмюровских орбит и несимметричных $\left(\left\langle r_{1}\right\rangle \sim\left\langle r_{2}\right\rangle / 3\right)$ для $Z^{2+} e^{-} e^{-}$-конфигурации (здесь $\langle r\rangle-$ радиальное среднее). Отметим, что именно последние состояния наблюдались в экспериментах по лазерному возбуждению атома бария [42].

В настоящей работе мы получили в рамках неполномерной теории квазиклассического квантования явные формулы (27) для “тетраридберговской” серии квазиклассических уровней энергии дважды возбужденных состояний ${ }^{3)}$ гелиеподобных атомов, в пределе при $\hbar \rightarrow 0$ отвечающих коррелированному движению электронов (с общим орбитальным моментом $\mathbf{L} \neq 0$ ) по боровской орбите.

В частности, для атома гелия $(Z=2)$ эта серия квазиклассических уровней энергии связанных состояний (в атомных единицах) имеет вид

$$
\begin{aligned}
E_{l, \nu}=- & 196\left(l+\frac{1}{2}+4 \sqrt{\frac{8}{7}}\left(\nu_{2}+\frac{1}{2}\right)+\right. \\
& \left.\left.+4 \sqrt{\frac{9}{7}}\left(\nu_{3}+\frac{1}{2}\right)+4 \sqrt{\frac{15}{14}}\left(\nu_{4}+\frac{1}{2}\right)\right)\right)^{-2}
\end{aligned}
$$

\footnotetext{
3) По-видимому, эти возбужденные долгоживущие состояния, также как и состояния, отвечающие устойчивым конфигурациям ленгмюровских орбит и орбит "frozen planet”, могут быть обнаружены в экспериментах по лазерному возбуждению гелиеподобных атомов.
} 
В таблице приведены численные значения энергетических уровней $-E_{l, \nu}$ в зависимости от $l$ и $\nu=\left(\nu_{2}, \nu_{3}, \nu_{4}\right)$.

\begin{tabular}{|c|c|c|c|c|c|c|c|c|}
\hline$-E_{l, \nu}$ & $l=2$ & $l=3$ & $l=4$ & $l=5$ & $l=10$ & $l=20$ & $l=30$ & $l=50$ \\
\hline$\nu_{j}=0$ & 2.4327 & 1.9694 & 1.6269 & 1.1366 & 0.6840 & 0.2693 & 0.1434 & 0.0604 \\
\hline$\nu_{j}=1$ & 0.4076 & 0.3728 & 0.3423 & 0.3154 & 0.2188 & 0.1229 & 0.0786 & 0.0401 \\
\hline$\nu_{j}=2$ & 0.1611 & 0.1522 & 0.1441 & 0.1356 & 0.1066 & 0.0701 & 0.0496 & 0.0285 \\
\hline
\end{tabular}

Отметим, что экстраполяция полученной формулы на нижние уровни дает значение основного состояния $\left(l=1, \nu_{j}=0\right)$, равное 3.0809 , что на $6 \%$ отличается от экспериментального значения, полученного в работе [47].

3.2. Оценка точности квазиклассического спектра. Используя стандартную технику [3], несложно показать, что асимптотические собственные значения $E_{l, \nu}(\hbar)$ приближают точные уровни $E_{l, \nu}^{\mathrm{ex}}(\hbar)$ в области больших квантовых чисел. Справедлива следующая оценка:

$$
\left|E_{l, \nu}^{\mathrm{ex}}(\hbar)-E_{l, \nu}(\hbar)\right| \leqslant 9 \sqrt{80} \frac{m e_{0}^{4}}{\hbar^{2} l^{\prime 2}}\left(\sum_{j=1}^{5} \frac{\nu_{j}}{\left|l^{\prime}\right|}\right)^{3 / 2} Z^{2} f(Z, \gamma),
$$

где $f(Z, \gamma)$ - ограниченная по $Z$ функция, $z \geqslant 2,|\gamma|<1$.

3.3. Параметры асимптотических разложений. Формальная процедура вычислений асимптотик по “постоянной” Планка $\hbar, \hbar \rightarrow 0$, подразумевает, что в рассматриваемой ситуации безразмерным параметром квазиклассических разложений (пропорциональным $\hbar$ ) является отношение длины волны де Бройля $\lambda$ вдоль равновесной окружности к характерным размерам системы $R=R\left(L_{z}\right): \lambda / R \sim 1 /\left|l^{\prime}\right|$, где $l^{\prime}$ - "коллективное" орбитальное квантовое число двух электронов. Другие безразмерные параметры разложения, $\xi_{j}(\hbar), j=1, \ldots, 5$, пропорциональные $\hbar^{1 / 2}$, характеризуют малые квантовые отклонения электронов вдоль трансверсальных направлений (вдоль $\rho, z$ и $\theta$ ) в окрестности классической траектории. Параметры $\xi_{j}(\hbar)$ определяются квантовыми числами $\nu_{j}, \nu_{j}=0,1,2, \ldots, j=1, \ldots, 5$, которые задают амплитуды этих колебаний:

для радиальных колебаний

$$
\xi_{1,2}(\hbar)=\left.\left.\frac{\sqrt{\left\langle(\rho-R(L))^{2}\right\rangle}}{R(L)}\right|_{L=l^{\prime} \hbar} \sim \sqrt{\frac{\hbar}{m \omega_{1,2}}} \sqrt{\nu_{1,2}+\frac{1}{2}} R(L)\right|_{L=l^{\prime} \hbar} \sim \sqrt{\frac{\nu_{1,2}}{\left|l^{\prime}\right|}} \ll 1,
$$

для аксиальных колебаний

$$
\xi_{3,4}(\hbar)=\left.\left.\frac{\sqrt{\left\langle z^{2}\right\rangle}}{R(L)}\right|_{L=l^{\prime} \hbar} \sim \sqrt{\frac{\hbar}{m \omega_{3,4}}} \sqrt{\nu_{3,4}+\frac{1}{2}} R(L)\right|_{L=l^{\prime} \hbar} \sim \sqrt{\frac{\nu_{3,4}}{\left|l^{\prime}\right|}} \ll 1,
$$

для фазовых колебаний

$$
\xi_{5}(\hbar)=\left.\frac{\sqrt{\langle\theta-\pi\rangle}}{\pi}\right|_{L=l^{\prime} \hbar} \sim \sqrt{\frac{\nu_{5}}{\left|l^{\prime}\right|}} .
$$


3.4. Тонкое расщепление уровней. Приведем, следуя работе [48], тонкую структуру квазиклассических уровней энергии (27) гелиеподобного атома, отвечающих боровской орбите. Величина расщепления $\Delta E^{\mathrm{s}-\mathrm{o}}$ этих уровней, связанная со спин-орбитальным взаимодействием, имеет вид (в ридберговских единицах)

$$
\Delta E^{\mathrm{s}-\mathrm{o}}=\xi \frac{\alpha^{2}}{32} \frac{(3-4 Z)(4 Z-1)^{3}}{|l|^{5}},
$$

где $\alpha$ - постоянная тонкой структуры $\left(\alpha=e_{0}^{2} / \hbar c\right), \xi= \pm 1,0,0,|l| \gg 1$. Эта квазиклассическая формула по порядку величины расщепления совпадает с известным результатом Бете и Солпитера, см. книгу [49] (формула (40.6)), где методом теории возмущений получено тонкое расщепление для иона гелия, когда по крайней мере один внешний электрон находится в сильно возбужденном состоянии. Отличие состоит в несколько иной зависимости величины расщепления от заряда ядра $Z$. Для этих же состояний величина расщепления $\Delta E^{\mathrm{s}-\mathrm{s}}$, связанная со спин-спиновым взаимодействием электронов, имеет вид

$$
\begin{aligned}
& \Delta E^{\mathrm{s}-\mathrm{s}}=A(\xi) \frac{\alpha^{2}}{16} \frac{(4 Z-1)^{3}}{|l|^{6}}, \quad \xi= \pm 1,0, \\
& A( \pm 1)=1, \quad A(0)=3 \text { для парагелия, } \quad A(0)=-5 \text { для ортогелия. }
\end{aligned}
$$

3.5. Сверхтонкая структура уровней. Структура фазовых траекторий гелиеподобного атома, в частности, структура его ОПР, весьма чувствительна к малым возмущениям гамильтониана (1), связанным с квадрупольным моментом ядра $\epsilon, \epsilon \sim 10^{-25} \mathrm{~cm}^{2}$. Влияние квадрупольного момента ядра можно при определенных условиях [37] эффективно учесть путем добавления к потенциалу (2) слагаемого $V_{\kappa}=e_{0}^{2} \epsilon\left(\left|q_{1}\right|^{-3}+\left|q_{2}\right|^{-3}\right)$. Интересно отметить следующее. Существует возмущенная боровская орбита (ОПР) радиуса $R(\epsilon) \rightarrow R(0), \epsilon \rightarrow 0$. Но наряду с ней вблизи основного уровня $E_{0}=-24.58$ эВ в достаточно узком интервале энергий $\Delta E$ (для $Z=2$, $\left.\epsilon \sim 10^{-25} \mathrm{~cm}^{2}, \Delta E \approx 3 \ni \mathrm{B}\right)$ при $E \in\left(E_{2}, E_{2}+\Delta E\right)$, где $E_{2}=-23$ эВ, существуют также "несимметричные" и нерезонансные ОПР с радиусом движения электронов $R_{1}^{\alpha}(\kappa)<R_{2}^{\alpha}(\kappa), \alpha=1,2$. Эти орбиты существуют при условии согласования $\epsilon$ и $L$ в области параметров, задаваемой неравенством $\tilde{\epsilon}>C\left|l^{\prime}\right|^{4}, \tilde{\epsilon}=\epsilon m_{e}^{2} e_{0}^{4} / \hbar^{4}, C \approx 0.0629$ (см. [20], где также приведены явные формулы для $R_{1,2}^{\alpha}(\epsilon), R(\epsilon)$ и частоты нормальных колебаний).

3.6. О снятии вырождения квазиклассического спектра. Как мы уже отмечали, боровская фазовая траектория $\Lambda^{1}(E)(34)$ является резонансной. Это приводит к тому, что соответствующая $\Lambda^{1}(E)$ спектральная серия $E_{l, \nu}(\hbar)(27)$ является асимптотически вырожденной. Асимптотическое вырождение по всей видимости снимается при построении высших по $\hbar, \hbar \rightarrow 0$, членов асимптотики. Напомним, что речь здесь идет о степенно́м по параметру $\hbar$ вырождении, а не об экспоненциально малом расщеплении собственных значений, обусловленном туннельными эффектами. Соответствующие поправки к асимптотически вырожденному спектру в случае резонансов возникают как из классической, так и квантовой механики. 
При этом ясно, что определение "классических" поправок связано с построением нормальных форм высокого (более, чем второго) порядка классического гамильтониана в окрестности резонансного инвариантного тора. "Квантовые" поправки обусловлены квантовой деформацией скобок Пуассона (см. подробнее в [10]).

Принципиально важный подготовительный шаг к построению "классической” части расщепления асимптотически вырожденного спектра в общей ситуации был проделан в работах [17], [18], где получены явные формулы для обобщенных переменных действие-угол (переменные типа действие-угол и переменные гармонического осциллятора) в полной окрестности устойчивого изотропного тора. Процедура построения таких канонических переменных, как оказалось, сводится к решению некоторой системы квадратных уравнений (подробнее об этом см. в [18]). Тем не менее в соответствующей неполномерной теории квазиклассического квантования инвариантных изотропных многообразий с комплексным ростком остается еще много неясных вопросов в случае наличия резонансов. С этой точки зрения значимая для квантовой физики модель атома гелия (рассмотренная в работе) является чрезвычайно интересным примером неинтегрируемой системы с богатым набором резонансных объектов, в принципе пригодных для квантования в рамках неполномерной теории. В частности, ясно, что для боровской резонансной траектории $\Lambda^{1}(E)$ величина классической части поправок $\Delta E_{\mathrm{cl}}$, снимающих асимптотическое вырождение в членах порядка $\hbar^{2}$, определяется квантованием нормальной формы Бирхгофа в окрестности уже не резонансной точки покоя $r^{*}\left(L_{z}\right)$ приведенного гамильтониана $H_{L_{z}}[28]$.

Наряду с боровскими резонансными траекториями, рассмотренными в данной работе, гамильтонова система (32) допускает и другие инвариантные резонансные объекты, например, двумерные торы $\Lambda^{2}(E)$ или трехмерные инвариантные подмногообразия, гомеоморфные трехмерной группе вращений, лежащие в окрестности так называемых ленгмюровских орбит (с общим орбитальным моментом $L=0$ ) [46]. Всем таким объектам также отвечают новые квазиклассические спектральные серии, асимпотически вырожденные в первом приближении по параметру $\hbar, \hbar \rightarrow 0$. Их построение и вычисление степенно́го по этому параметру расщепления в этих спектральных сериях, порожденного резонансными эффектами, составляет предмет дополнительных исследований, которые будут представлены в последующих публикациях. В заключение приведем лишь простейший пример однопараметрического семейства двумерных торов $\Lambda^{2}(E)$, порожденных боровскими резонансными кривыми $\Lambda^{1}(E)$. В декартовых координатах боровская фазовая траектория двух электронов $\Lambda^{1}(E)$ задается формулами

$$
\Lambda^{1}(E)=(P=m \dot{Q}, Q), \quad Q=\left(R \cos \omega_{0} t, R \sin \omega_{0} t, 0,-R \cos \omega_{0} t,-R \sin \omega_{0} t, 0\right),
$$

где радиус $R=R(E)$ и частота $\omega_{0}=\omega_{0}(E)$ определены формулами (35). Пусть $L_{1}$ - проекция полного орбитального момента двух электронов на ось $x, L_{1}=$ $y_{1} p_{z_{1}}-z_{1} p_{y_{1}}+y_{2} p_{z_{2}}-z_{2} p_{y_{2}}=L_{x_{1}}+L_{x_{2}}$. Траектории гамильтониана $L_{1}$ являются $2 \pi$-периодическими по $\tau$, где $\tau$ - "время" сдвига вдоль траекторий этого гамильтониана. Действие фазового потока $g_{L_{1}}^{\tau}$ на точки кривой $\Lambda^{1}(E)$ порождает двумерный 
изотропный тор $\Lambda^{2}(E)=\bigcup_{0 \leqslant \tau \leqslant 2 \pi} g_{L_{1}}^{\tau} \Lambda^{1}(E)$, очевидно, инвариантный относительно исходного гамильтониана $\left(\left\{H, L_{1}\right\}=0\right)$. Проекция $\pi_{q} \Lambda^{2}(E)$ тора $\Lambda^{2}(E)$ на конфигурационное пространство $\mathbb{R}_{q}^{6}$ есть двумерная сфера $S^{2}(E)$ радиуса $R(E)$, которая задается формулами

$$
\begin{array}{lll}
x_{1}=R \cos \omega_{0} t, & y_{1}=R \sin \omega_{0} t \cos \tau, & z_{1}=-R \sin \omega_{0} t \sin \tau, \\
x_{2}=-R \cos \omega_{0} t, & y_{2}=-R \sin \omega_{0} t \sin \tau, & z_{2}=R \sin \omega_{0} t \sin \tau,
\end{array}
$$

где $\varphi=\omega_{0} t(\bmod 2 \pi), \tau(\bmod 2 \pi)$ - угловые координаты на торе $\Lambda^{2}(E)$. В полюсах этой сферы $(\varphi=0, \tau=0)$ отображение проекции $\pi_{q}: \Lambda^{2} \rightarrow \mathbb{R}_{q}^{6}$ является вырожденным и имеет ранг 1.

Квазиклассическое квантование этих объектов в рамках теории, изложенной в работах [7]-[9], является темой дополнительного исследования.

Благодарности. Авторы выражают благодарность И. В. Тютину за полезные обсуждения и ценные замечания, а также студентам МФТИ А. Ю. Чаплыгину и М.Н. Дмитриеву за помощь в вычислениях констант при оценке точности квазиклассического спектра. Работа выполнена при поддержке РФФИ (грант № 05-0100968).

\section{Список литературы}

[1] N. Bohr, Phil. Mag., 26 (1913), 1; 476; 857.

[2] G. Tanner, K. Richter, J. Rost, Rev. Mod. Phys., 72 (2000), 497.

[3] В. П. Маслов, Теория возмущений и асимптотические методы, Изд-во МГУ, М., 1965.

[4] В.П. Маслов, М.В. Федорюк, Квазиклассическое приближение для уравнений квантовой механики, Наука, М., 1976.

[5] Ж. Лере, Лагранжев анализ и квантовая механика. Математическая структура, связанная с асимптотическими разложениями и индексом Маслова, Мир, М., 1981.

[6] В. И. Арнольд, Функи. анализ и его прилож., 6:2 (1972), 12.

[7] В. П. Маслов, Комплексный метод ВКБ в нелинейных уравнениях, Наука, М., 1977.

[8] В. В. Белов, С. Ю. Доброхотов, ДАН СССР. Сер. матем., 298:5 (1988), 1037.

[9] В. В. Белов, С. Ю. Доброхотов, ТМФ, 92:2 (1992), 215.

[10] В. В. Белов, О. С. Доброхотов, С. Ю. Доброхотов, Матем. заметки, 69:4 (2001), 483.

[11] G. Wentzel, Z. Phys., 38 (1926), 518.

[12] H. A. Kramers, Z. Phys., 13 (1923), 312.

[13] L. Brillouin, C. R. Acad. Sci. Paris, 183 (1926), 24.

[14] В. М. Бабич, Зап. научн. сем. ЛОМИ, 9 (1968), 15.

[15] J. V. Ralston, Comm. Math. Phys., 51 (1976), 219.

[16] С. Ю. Доброхотов, Н. Н. Нехорошев, Б. Валиньо, УМН, 39:3 (1984), 233.

[17] В. Белов, С. Доброхотов, В. Максимов, Докл. РАН, 381 (2001), 452.

[18] В. Белов, С. Доброхотов, В. Максимов, ТМФ, 135:3 (2003), 378.

[19] В. В. Козлов, Симметрии, топология и резонансы в гамильтоновой механике, Изд-во УГУ, Ижевск, 1995.

[20] В. В. Белов, В. А. Максимов, Матем. заметки, 64:2 (1998), 297.

[21] V. V. Belov, V. A. Maksimov, Russ. J. Math. Phys., 7 (2000), 363.

[22] В. В. Белов, С. Ю. Доброхотов, В. М. Оливе, Докл. РАН. Сер. физ., 38:7 (1993), 263.

[23] S. Yu. Dobrokhotov, V. Martínez-Olivé, A. I. Shafarevich, Russ. J. Math. Phys., 3 (1995), 133.

[24] V. V. Belov, J. L. Volkova, Russ. J. Math. Phys., 1 (1993), 409. 
[25] V. Belov, V. Olivé, J. Volkova, Phys. A, 28 (1995), 5799; 1995, 5811.

[26] С. Доброхотов, В. Мартинес-Оливе, Тр. ММО, 58 (1997), 3.

[27] А. В. Болсинов, А. Т. Фоменко, Введение в топологию интегрируемых гамильтоновых систем, Наука, М., 1997.

[28] В. И. Арнольд, Математические методы классической механики, Наука, М., 1983.

[29] A. Einstein, Verh. Dtsch. Phys. Ges., 19 (1917), 82.

[30] J. Keller, Ann. Phys., 4 (1958), 180.

[31] A. Sommerfeld, Ann. Phys., 356:17 (1916), 1; 356:18 (1916), 125.

[32] A. Voros, "Exact anharmonic quantization condition (in one dimension)", Quasiclassical Methods (Minneapolis, MN, USA, 1995), IMA Vol. Math. Appl., 95, eds. J. Rauch, B. Simon, Springer, New York, 1997, 189.

[33] A. Voros, "The WKB-Maslov method for nonseparable systems", Géométrie symplectique et physique mathematique (Aix-en-Provence, 1974), ed. J.-M. Souriau, Éditions du CNRS, Paris, 1975, 277.

[34] С. Ю. Доброхотов, А.И. Шафаревич, "Квазиклассическое квантование изотропных многообразий гамильтоновых систем", Топологические методы в теории гамильтоновых систем, ред. А.В. Болсинов, А. Т. Фоменко, А.И. Шафаревич, Факториал, M., 1998, 41; S. Yu. Dobrokhotov, A.I. Shafarevich, Russ. J. Math. Phys., 5 (1997), 267; М.В. Карасев, Квантовая редукиия на орбиты алгебр симметрий и задача Эренфеста, Препринт ИТФ-87-157Р, ИТФ АН УССР, Киев, 1988; М. V. Karasev, Yu. M. Vorob'ev, "Adapted connections, Hamiltonian dynamics, geometric phases, and quantization over isotropic submanifolds", Coherent Transform, Quantization and Poisson Geometry, Amer. Math. Soc. Transl. Ser. 2, 187, ed. M. V. Karasev, Amer. Math. Soc., Providence, RI, 1998, 203.

[35] J. E. Marsden, A. Weinstein, Rep. Math. Phys., 5 (1974), 121.

[36] В. В. Белов, Е. М. Воробьев, Сборник задач по дополнительным главам математической физики, Высшая школа, М., 1978.

[37] И. И. Собельман, Введение в теорию атомных спектров, ГИФМЛ, М., 1968.

[38] I. Langmuir, Phys. Rev., 17 (1921), 339.

[39] U. Fano, Rep. Prog. Phys., 46 (1983), 97; D. Wintgen, A. Bürgers, K. Richter, G. Tanner, Progr. Theor. Phys. Suppl., 116 (1994), 121; J. A. West, Z. D. Gaeta, C. R. Stroud, Jr., Phys. Rev. A, 58:1 (1998), 186.

[40] J. Müller, J. Burgdörfer, Phys. Rev. Lett., 70:16 (1993), 2375; J. Müller, X. Yang, J. Burgdörfer, Phys. Rev. A, 49:4 (1994), 2470.

[41] K. Richter, D. Wintgen, Phys. Rev. Lett., 65:15 (1990), 1965; F. Benvenuto, G. Casati, D. L. Shepelyansky, Phys. Rev. A, 53 (1996), 737.

[42] U. Eichmann, V. Lange, W. Sandner, Phys. Rev. Lett., 64 (1990), 274; 68 (1992), 21.

[43] K. Richter, D. Wintgen, J. Phys. B, 23 (1990), L197.

[44] K. Richter, D. Wintgen, J. Phys. B, 24 (1991), L565.

[45] M. C. Gutzwiller, Chaos in Classical and Quantum Mechanics, Springer, New York, 1990.

[46] A. Bürgers, D. Wintgen, J. Phys. B, 27 (1994), L131; J. Müller, J. Burgdörfer, D. Noid, Phys. Rev. A, 45:3 (1992), 1471; P. Gaspard, S. A. Rice, Phys. Rev. A, 48:1 (1993), 54.

[47] S. D. Bergeson, A. Balakrishnan, K. G. H. Baldwin et al., Phys. Rev. Lett., 80 (1998), 3475.

[48] В. В. Белов, В. А. Максимов, ТМФ, 126:3 (2001), 455.

[49] Г. Бете, Э. Солпитер, Квантовая механика атомов с одним и двумя электронами, Физматгиз, М., 1969. 